\title{
High Yield Selective Acylation of Polyamines: Proton as Protecting Group
}

Asmik Oganesyan, Iris A. Cruz, Roberto B. Amador, Nohemy A. Sorto, Jose Lozano, Carlos E. Godinez, Jaime Anguiano, Heather Pace, Ghiwa Sabih, and Carlos G. Gutierrez*

Department of Chemistry \& Biochemistry, California State University, Los Angeles, CA 90032-8202

\section{cgutier@calstatela.edu}

Supporting Information: Experimental procedures and physical data (pages S-1-8) for 4-6, 8-10, 12, 13, 15, 16, 18, 19, 21, and 23, and copies of ${ }^{1} \mathrm{H}$ NMR, ${ }^{13} \mathrm{C}$ NMR and IR spectra (pages S-10-49; ${ }^{1} \mathrm{H}$ NMR and IR only for $\mathbf{9}$ ).

General Methods. Reactions were conducted in oven-dried $\left(120{ }^{\circ} \mathrm{C}\right)$ glassware under a positive dry-nitrogen atmosphere and were magnetically stirred. Reagents obtained from commercial suppliers were used without purification unless otherwise noted. The synthesis of 1,3,5,7-tetra(aminomethyl)adamantane (1) was reported previously. ${ }^{6}$ Solvents were distilled over drying agents. ${ }^{16}$ Thin layer chromatography (TLC) was performed on glass plates pre-coated with $0.25 \mathrm{~mm} 60 \AA \mathrm{F}_{254}$ silica gel (Whatman). Purification of crude products was done by flash chromatography on $60 \AA 230-400$ mesh silica gel (Fisher Scientific). ${ }^{1} \mathrm{H}$ NMR and proton-decoupled ${ }^{13} \mathrm{C}$ NMR spectra were obtained in $\mathrm{CDCl}_{3}$ and DMSO- $d_{6}$ solutions using Bruker FT-NMR spectrometers operating at either 300,400 , or $600 \mathrm{MHz}$, with spectra referenced to residual solvent protons and carbons, respectively. Data for ${ }^{1} \mathrm{H}$ NMR spectra are reported as follows: chemical shift, multiplicity $(\mathrm{s}=$ singlet, $\mathrm{d}=$ doublet, $\mathrm{t}=$ triplet, $\mathrm{q}=$ quartet, $\mathrm{m}=$ multiplet, $\mathrm{br}=$ broad), coupling constant $(\mathrm{Hz})$, and integration. Infrared spectra were recorded on a Perkin Elmer Spectrum 100 FT-IR instrument. High-resolution mass spectra (HRMS) were recorded at the mass spectrometry facility at the California Institute of Technology on a JEOL MSRoute instrument. All references are numbered as presented in the manuscript; additional references on page S-8 of the supporting information section.

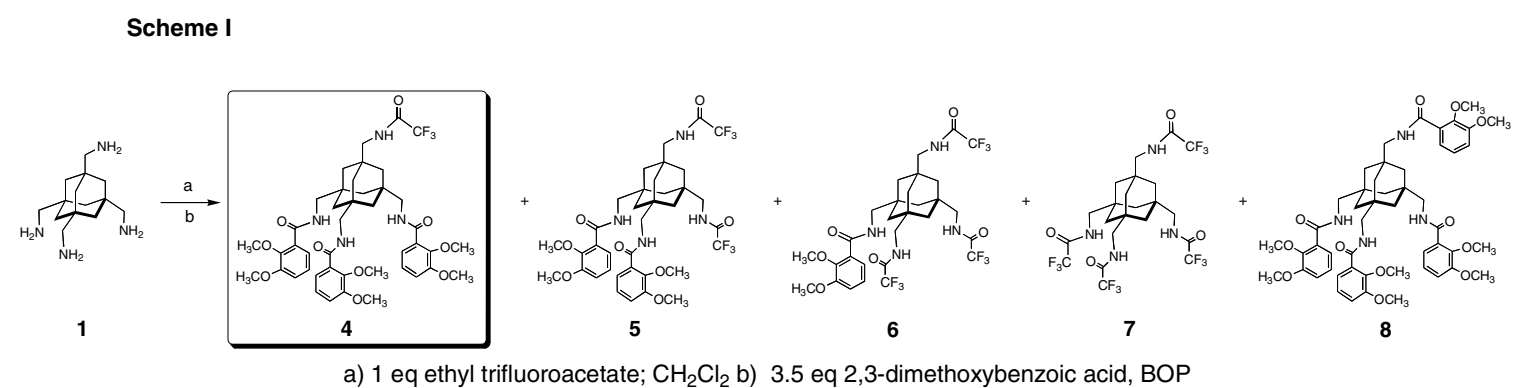

1- $N$-Trifluoroacetylaminomethyl-3,5,7-tri( $N$-2,3-dimethoxybenzoylaminomethyl)adamantane (4): A solution of $0.252 \mathrm{~mL} 1.01 \mathrm{M} t$-BuOK $(0.252 \mathrm{mmol})$ in THF was diluted with $10 \mathrm{~mL}$ methanol, and added dropwise over $1 \mathrm{~h}$ (syringe pump) to a second solution of $0.100 \mathrm{~g} \quad(0.252 \mathrm{mmol}) \quad$ 1,3,5,7-tetra(aminomethyl)adamantane 
tetrahydrochloride $(\mathbf{1} \mathbf{\bullet} \mathbf{H C l})$ in $100 \mathrm{~mL}$ methanol. After $3 \mathrm{~h}$, the reaction mixture was cooled down in an ice bath and ethyl trifluoroacetate $(0.030 \mathrm{~mL}, 0.252 \mathrm{mmol})$ in $20 \mathrm{~mL}$ THF was added via syringe pump. The mixture was allowed to warm to room temperature, stirred overnight, and reacted with $1.521 \mathrm{~mL} 1.01 \mathrm{M} t$-BuOK $(1.521 \mathrm{mmol})$. The mixture was stirred for $10 \mathrm{~min}$ before treating it with a solution of 2,3dimethoxybenzoic acid $(0.142 \mathrm{~g}, 0.781 \mathrm{mmol})$ activated by BOP reagent $(0.367 \mathrm{~g}, 0.832$ mmol $)^{8}$ and triethylamine (TEA) $(0.109 \mathrm{~mL}, 0.781 \mathrm{mmol})$ in $20 \mathrm{~mL}$ dichloromethane. The mixture was stirred for $15 \mathrm{~h}$ and the solvent was evaporated. The crude product was washed with $20 \mathrm{~mL}$ EtOAc, gravity filtered, and concentrated. The crude mixture was purified by column chromatography with gradient mixtures of hexanes and ethyl acetate (10-75\%), which eluted products in the order: $0.194 \mathrm{~g}$ mono(trifluoroacetamide) 4 (92\%) (see characterization below) and $0.004 \mathrm{~g}$ tetracatecholamide 8 (2\%). When TEA was used as the base for initial monodeprotonation, the product distribution was as follows: $0.002 \mathrm{~g}$ tris(trifluoroacetamide) $\mathbf{6}(2 \%), 0.016 \mathrm{~g}$ bis(trifluoroacetamide) $\mathbf{5}(12 \%), 0.146 \mathrm{~g}$ $4(69 \%)$, and $0.015 \mathrm{~g} 8(5 \%)$.

Characterization of $4:{ }^{1} \mathrm{H}$ NMR $\left(300 \mathrm{MHz}, \mathrm{CDCl}_{3}\right) \delta 1.25-1.49(\mathrm{~m}, 11 \mathrm{H}), 2.01(\mathrm{~s}, 1 \mathrm{H})$, $3.13(\mathrm{~d}, J=7 \mathrm{~Hz}, 2 \mathrm{H}), 3.27$ (d, $J=6 \mathrm{~Hz}, 6 \mathrm{H}), 3.84(\mathrm{~s}, 9 \mathrm{H}), 3.86(\mathrm{~s}, 9 \mathrm{H}), 7.01(\mathrm{~d}, J=$ $3 \mathrm{~Hz}, 3 \mathrm{H}), 7.11(\mathrm{t}, J=1 \mathrm{~Hz}, 3 \mathrm{H}), 7.19(\mathrm{t}, J=1 \mathrm{~Hz}, 3 \mathrm{H}), 7.62(\mathrm{~d}, J=7 \mathrm{~Hz}, 3 \mathrm{H}), 8.08$ $(\mathrm{m}, J=7 \mathrm{~Hz}, 3 \mathrm{H}) ;{ }^{13} \mathrm{C} \mathrm{NMR}\left(100 \mathrm{MHz}, \mathrm{CDCl}_{3}\right) \delta 36.05,39.29,39.55,41.31,42.01$, 42.30, 42.88, 49.43, 50.10, 50.67, 56.12, 60.28, 61.39, 62.38, 76.68, 76.99, 77.32, 77.96, $78.24,115.48,115.68,117.45,122.89,122.96,123.87,124.29,124.79,126.58,126.85$, 147.50, 152.57, 157.73 (q, ${ }^{2} J=15 \mathrm{~Hz}, \mathrm{COCF}_{3}$ ), 165.37; IR (solid): 3341, 3246, 3078, 2922, 2846, 1712, 1644, 1575, 1535, 1421, 1309, 1267, 1201, 1186, 1151, 1062, 982, $802,756 \mathrm{~cm}^{-1}$; HRMS-FAB (m/z): [M+H] $]^{+}$calcd 841.3665; found 841.3639.

\section{1,3-Di(trifluoroacetyaminomethyl)-5,7-di( $N$-2,3-dimethoxybenzoylaminomethyl)-}

adamantane (5): ${ }^{1} \mathrm{H}$ NMR $\left(300 \mathrm{MHz}, \mathrm{CDCl}_{3}\right) \delta 1.28\left(\mathrm{~m}, 12 \mathrm{H}\right.$, endo- $\left.\mathrm{CH}_{2}\right) 3.12(\mathrm{~d}, J=7$ $\mathrm{Hz}, 4 \mathrm{H}$, exo- $\left.\mathrm{CH}_{2}\right), 3.26$ (d, $J=4 \mathrm{~Hz}, 4 \mathrm{H}$, exo- $\left.\mathrm{CH}_{2}\right), 3.86$ (s, $\left.6 \mathrm{H}, m-\mathrm{OCH}_{3}\right), 3.87$ (s, 6 $\left.\mathrm{H}, o-\mathrm{OCH}_{3}\right), 7.01$ (overlapping m, $\left.2 \mathrm{H}, \mathrm{ArH}\right), 7.13(\mathrm{~m}, 4 \mathrm{H}), 7.61(\mathrm{~d}, J=6 \mathrm{~Hz}, 2 \mathrm{H}), 8.12$ $(\mathrm{m}, 2 \mathrm{H},-\mathrm{NH}) ;{ }^{13} \mathrm{C}$ NMR $\left(100 \mathrm{MHz}, \mathrm{CDCl}_{3}\right) \delta 29.65,35.32,36.10,36.20,39.52,39.63$, $40.77,41.25,41.49,41.79,42.07,42.25,49.28,49.37,49.95,50.05,50.40,50.57,56.07$, $61.45,36.33,36.46,42.12,50.25,56.39,61.66,116.22,115.99,123.10,124.66,126.70$, 147.74, 152.86, $157.36\left(\mathrm{q},{ }^{2} J=15 \mathrm{~Hz}, C \mathrm{COCF}_{3}\right), 166.10(C \mathrm{OAr})$; IR $\left(\mathrm{CDCl}_{3}\right): 3372,3271$, 3073, 2921, 2846, 1712, 1649, 1575, 1537, 1472, 1462, 1426, 1307, 1264, 1209, 1183, 1156, 1067, 991, 905, 807, $728 \mathrm{~cm}^{-1}$; HRMS-FAB (m/z): $[\mathrm{M}+\mathrm{H}]^{+}$calcd 773.2985; found 773.2977.

\section{3,5-Tri( $N$-trifluoroacetylaminomethyl)-7-( $N$-2,3-dimethoxybenzoylaminomethyl)-}

adamantane (6): ${ }^{1} \mathrm{H}$ NMR $\left(300 \mathrm{MHz}, \mathrm{CDCl}_{3}\right) \delta 1.15-1.30\left(\mathrm{~m}, 12 \mathrm{H}\right.$, endo- $\left.\mathrm{CH}_{2}\right), 3.14(\mathrm{~d}$, $J=7 \mathrm{~Hz}, 6 \mathrm{H}$, exo- $\left.\mathrm{CH}_{2}\right), 3.26\left(\mathrm{~d}, J=6 \mathrm{~Hz}, 2 \mathrm{H}\right.$, exo- $\left.\mathrm{CH}_{2}\right) ; 3.89\left(\mathrm{~s}, 3 \mathrm{H}, m-\mathrm{OCH}_{3}\right), 3.91$ (s, $\left.3 \mathrm{H}, o-\mathrm{OCH}_{3}\right), 6.79(\mathrm{t}, J=6 \mathrm{~Hz}, 1 \mathrm{H}) ; 7.05$ (overlapping m, $\left.4 \mathrm{H}, \mathrm{ArH},-\mathrm{NH}\right), 7.59$ (d, $J$ $=6 \mathrm{~Hz}, 1 \mathrm{H}, o-\mathrm{ArH}), 8.19(\mathrm{t}, J=6 \mathrm{~Hz}, 1 \mathrm{H}) ;{ }^{13} \mathrm{C} \mathrm{NMR}\left(75 \mathrm{MHz}, \mathrm{CDCl}_{3}\right) \delta 38.96,39.39$, $39.71,40.45,40.74,41.49,42.02,42.24,42.44,49.32$, 49.91, 50.33, 50.62, 51.03, 56.18, $56.89,61.46,62.15,77.69,78.01,79.04,114.52,115.67,116.60,117.39,118.07,120.24$, $122.71,123.45,124.53,125.23,126.27,130.84,147.51,152.68,157.52,164.52$; IR 
$\left(\mathrm{CDCl}_{3}\right)$ 3352, 2916, 2846, 1717, 1646, 1578, 1535, 1459, 1264, 1209, 1163, 1062, 996 $\mathrm{cm}^{-1}$; HRMS-FAB $(\mathrm{m} / \mathrm{z}):[\mathrm{M}+\mathrm{H}]^{+}$calcd 705.2334; found 705.2346

1,3,5,7-Tetra( $N$-2,3- dimethoxybenzoylaminomethyl)adamantane (8): ${ }^{1} \mathrm{H}$ NMR (300 $\left.\mathrm{MHz}, \mathrm{CDCl}_{3}\right) \delta 1.34(\mathrm{~s}, 12 \mathrm{H}), 3.27(\mathrm{~d}, J=6 \mathrm{~Hz}, 8 \mathrm{H}) ; 3.81(\mathrm{~s}, 12 \mathrm{H}), 3.84(\mathrm{~s}, 12 \mathrm{H}), 7.00$ (d, $J=4 \mathrm{~Hz}, 4 \mathrm{H}), 7.09(\mathrm{t}, J=2 \mathrm{~Hz}, 4 \mathrm{H}), 7.63(\mathrm{~d}, J=6 \mathrm{~Hz}, 1 \mathrm{H}), 8.05(\mathrm{t}, J=11 \mathrm{~Hz}, 4 \mathrm{H})$; ${ }^{13} \mathrm{C}$ NMR $\left(100 \mathrm{MHz}, \mathrm{CDCl}_{3}\right) \delta 36.04,42.54,50.29,56.21,61.47,115.69,123.15,124.29$, 126.83, 147.63, 152.63, 165.44; IR (solid) 3367, 3195, 2932, 2841, 1719, 1648, 1573, 1530, 1467, 1307, 1264, 1055, 986, 804, $749 \mathrm{~cm}^{-1}$; HRMS-FAB (m/z): $[\mathrm{M}+\mathrm{H}]^{+}$calcd 909.4286; found 909.4282 .

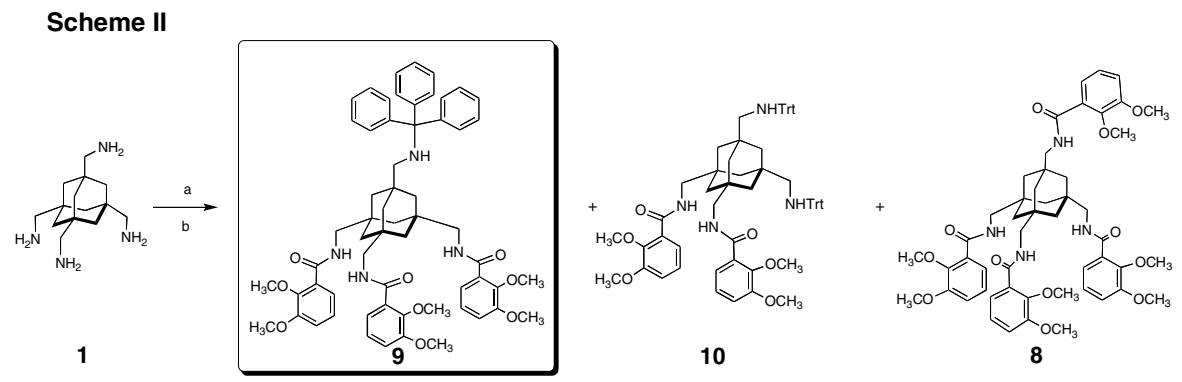

a) 1 eq trityl chloride, $\mathrm{CH}_{2} \mathrm{Cl}_{2}$; b) 3.1 eq 2,3-dimethoxybenzoyl chloride

1- $\mathrm{N}$-Tritylaminomethyl-3,5,7-tri( $\mathrm{N}$-2,3-dimethoxybenzoylamidomethyl)adamantane (9): A solution of tetraamine $1(0.238 \mathrm{~g}, 0.944 \mathrm{mmol})$ in $100 \mathrm{~mL} \mathrm{CH} \mathrm{Cl}_{2}$ was cooled down in an ice bath. Trityl chloride $(0.236 \mathrm{~g}, 0.944 \mathrm{mmol})$ in $10 \mathrm{~mL} \mathrm{CH}_{2} \mathrm{Cl}_{2}$ was added dropwise via syringe pump over $1 \mathrm{~h}$. The mixture was allowed to warm to room temperature, stirred for $3 \mathrm{~h}$, and reacted with $0.394 \mathrm{~mL}(2.823 \mathrm{mmol})$ of triethylamine. The resulting mixture was reacted with a solution of 2,3-dimethoxybenzoyl chloride $(0.625 \mathrm{~g}, 3.110 \mathrm{mmol})$ in $8 \mathrm{~mL} \mathrm{CH}_{2} \mathrm{Cl}_{2}$. The mixture was rotary evaporated and the residue taken up in EtOAc, and filtered. The filtrate was concentrated under vacuum and the residue purified by column chromatography with gradient mixtures of ethyl acetate (10-100\%) and hexanes, which eluted: $0.030 \mathrm{~g}$ ditrityl $10(6 \%) ; 0.373$ g monotrityl 9 (40\%), and $0.043 \mathrm{~g}$ tetracatecholamide $8(5 \%)$. Characterization of 9: ${ }^{1} \mathrm{H}$ NMR (600 $\left.\mathrm{MHz}, \mathrm{CDCl}_{3}\right) \delta 1.24(\mathrm{~m}, 13 \mathrm{H}), 3.31(\mathrm{~d}, J=5 \mathrm{~Hz}, 6 \mathrm{H}), 3.83(\mathrm{~s}, 9 \mathrm{H}), 3.84-3.90(\mathrm{~m}, 20$ $\mathrm{H}), 7.04-7.25(\mathrm{~m}, 15 \mathrm{H}), 7.44(\mathrm{~m}, 6 \mathrm{H}), 7.68(\mathrm{~d}, J=3 \mathrm{~Hz}, 3 \mathrm{H}), 8.03(\mathrm{t}, J=5 \mathrm{H}, 3 \mathrm{H}) ;{ }^{13} \mathrm{C}$ NMR $\left(100 \mathrm{MHz}, \mathrm{CDCl}_{3}\right) \delta 29.17,29.34,29.72,31.91,35.60,35.94,39.62,41.37,42.72$, $43.02,49.49,50.38,54.75,56.17,61.54,62.16,70.55,115.46,123.06,124.43,126.22$, 126.90, 129.36, 130.06, 146.04, 147.46, 152.61, 165.44; IR $\left(\mathrm{CDCl}_{3}\right) 3377,2927,2846$, 1742 (EtOAc), 1654, 1575, 1530, 1456, 1426, 1259, 1219, 1062, 989, 905, $807 \mathrm{~cm}^{-1}$; HRMS-FAB (m/z): $[\mathrm{M}+\mathrm{H}]^{+}$calcd 987.4908; found 987.4910.

1,3-di( $N$-Tritylaminomethyl)- 5,7-di( $N$-2,3-dimethoxybenzoylaminomethyl)adamantane (10): ${ }^{1} \mathrm{H}$ NMR $\left(600 \mathrm{MHz}, \mathrm{CDCl}_{3}\right) \delta 1.26-1.90(\mathrm{~m}, 12 \mathrm{H}), 3.30(\mathrm{~d}, J=7 \mathrm{~Hz}$, 4H), $3.83(\mathrm{~s}, 6 \mathrm{H}), 3.90-3.92(\mathrm{~m}, 10 \mathrm{H}), 7.04-7.25(\mathrm{~m}, 34 \mathrm{H}), 7.68(\mathrm{~d}, J=7 \mathrm{~Hz}, 2 \mathrm{H}), 8.03$ $(\mathrm{t}, J=6 \mathrm{H}, 2 \mathrm{H})$; IR $\left(\mathrm{CDCl}_{3}\right) 3362,3301,2911,2841,1646,1573,1535,1472,1360$, 1264, 1072, $991 \mathrm{~cm}^{1}$; HRMS-FAB (m/z): $[\mathrm{M}+\mathrm{H}]^{+}$calcd 1066.3438; found 1066.6080. 


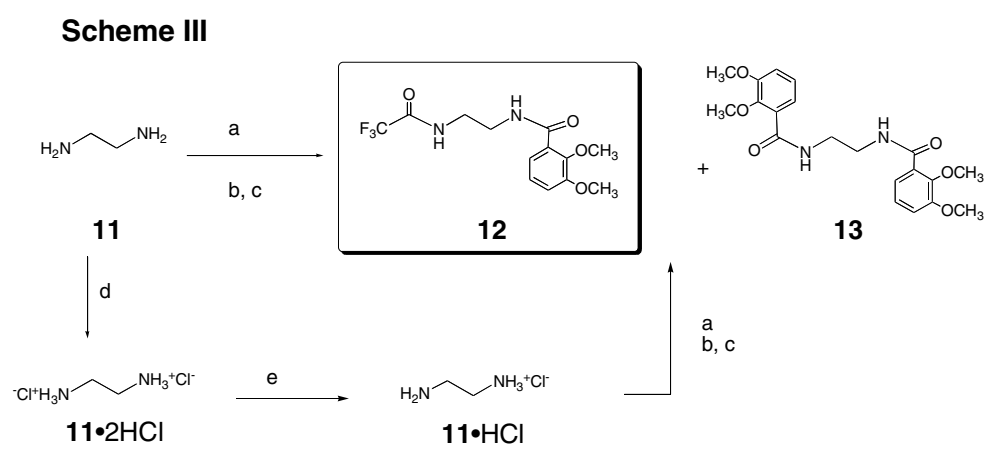

a) 1 eq ethyl triflouroacetate; b) 1.1 eq 2, 3-dimethoxybenzoyl chloride; c) excess $\mathrm{Et}_{3} \mathrm{~N}$; d) $2 \mathrm{HCl}$; e) 1 eq $\mathrm{NaOCH}_{3}$

1- $N$-(2,3-dimethoxybenzoyl)-2- $N$-(trifluoroacetyl)-1,2-ethylenediamine (12) from neutral ethylenediamine: A solution of $0.252 \mathrm{~mL}(3.761 \mathrm{mmol})$ ethylene diamine in $150 \mathrm{~mL}$ dry $\mathrm{CH}_{2} \mathrm{Cl}_{2}$ was cooled down in an ice bath and reacted through dropwise addition (syringe pump) of $0.448 \mathrm{~mL}$ trifluoroethyl acetate $\left(3.761 \mathrm{mmol}\right.$ ) in $4 \mathrm{~mL} \mathrm{CH}_{2} \mathrm{Cl}_{2}$ over $1 \mathrm{~h}$. The mixture was allowed to warm to room temperature and stirred for $1.5 \mathrm{~h}$, and then reacted with a solution of $0.752 \mathrm{~g}(4.136 \mathrm{mmol})$ 2,3-dimethoxy benzoic acid, 1.828 g BOP (4.136 mmol), and $2.092 \mathrm{~mL}$ TEA (15.044 mmol) in $25 \mathrm{~mL}$ of $\mathrm{CH}_{2} \mathrm{Cl}_{2}$. The mixture was stirred for $15 \mathrm{~min}$, rotary evaporated, and taken into $50 \mathrm{~mL}$ EtOAc. Triethylammonium salts were removed by gravity filtration; the filtrate was rotary evaporated and the residue was purified by column chromatography with gradients of EtOAc (10-75\%) and hexanes to elute $0.361 \mathrm{~g} 12$ (30\%) and $0.453 \mathrm{~g} 13$ (62\%) as white solids.

$N$-2,3-dimethoxybenzoyl-2- $N$-(trifluoroacetyl)ethylenediamine

from diammonium salt 11.2HCl: A solution of $0.500 \mathrm{~g}(3.76 \mathrm{mmol})$ ethylenediamine dihydrochloride in $100 \mathrm{~mL}$ DMSO was treated with dropwise addition (syringe pump) of $3.76 \mathrm{~mL}(3.76 \mathrm{mmol}) 1.01 \mathrm{M} t$-BuOK in THF over $1 \mathrm{~h}$. After stirring the mixture an additional $2 \mathrm{~h}$, a solution of $0.448 \mathrm{~mL}$ of ethyl trifluoroacetate $(3.76 \mathrm{mmol})$ in $10 \mathrm{~mL}$ $\mathrm{CH}_{2} \mathrm{Cl}_{2}$ was added dropwise via syringe pump. The mixture was stirred at room temperature for $2 \mathrm{~h}$ before reacting it with a solution of $752 \mathrm{~g}(3.76 \mathrm{mmol}) 2,3-$ dimethoxybenzoic acid, $1.822 \mathrm{~g} \mathrm{BOP}(4.14 \mathrm{mmol})$ and $2.092 \mathrm{~mL}$ TEA $(15.04 \mathrm{mmol})$ in $20 \mathrm{~mL}$ DMSO. The mixture was stirred overnight, extracted with EtOAc $(3 \times 50 \mathrm{~mL})$. The organic phased was washed with distilled water, brine, dried over $\mathrm{MgSO}_{4}$, and concentrated to afford a yellow oil. The product was further purified by column chromatography using gradient mixtures of EtOAc (10-75\%) and hexanes to afford 1.010 g $12(84 \%)$ and $0.066 \mathrm{~g}$ biscatecholamide $13(9 \%)$.

Characterization of 12: ${ }^{1} \mathrm{H} \mathrm{NMR}\left(600 \mathrm{MHz}, \mathrm{CDCl}_{3}\right) \delta 3.57(\mathrm{dt}, J=11 \mathrm{~Hz}, 5 \mathrm{~Hz}, 2 \mathrm{H})$, $3.68(\mathrm{dt}, J=10 \mathrm{~Hz}, 5 \mathrm{~Hz}, 2 \mathrm{H}), 3.89(\mathrm{~s}, 3 \mathrm{H}), 3.91(\mathrm{~s}, 3 \mathrm{H}), 7.07$ (d, $J=2 \mathrm{~Hz}, 1 \mathrm{H}), 7.16(\mathrm{t}$, $J=4 \mathrm{~Hz}, 1 \mathrm{H}), 7.66(\mathrm{~d}, J=2 \mathrm{~Hz}, 1 \mathrm{H}), 8.35(\mathrm{t}, J=7 \mathrm{~Hz}, 1 \mathrm{H}), 8.45(\mathrm{t}, J=12 \mathrm{~Hz}, 1 \mathrm{H})$;

${ }^{13} \mathrm{C}$ NMR (100 MHz, DMSO- $\left.d_{6}\right) \delta 55.96,60.81,111.60,114.46,114.99,117.33,120.19$, $120.80,123.85,124.28,125.12,129.32$, 146.54, 152.502, 155.96, 156.33, 156.68, 157.05, 165.71; IR (solid) 3362, 3251, 3084, 2952, 1707, 1651, 1560, 1520, 1181, 1146, 1065 , $981 \mathrm{~cm}^{-1}$; HRMS-FAB (m/z): $[\mathrm{M}]^{+}$calcd 320.0984; found; $20.0982 ; \mathrm{mp} 140-141{ }^{\circ} \mathrm{C}$ : 
1,2- $N, N$ '-di(2,3-dimethoxybenzoyl)-1,2-ethylenediamine (13): ${ }^{1} \mathrm{H}$ NMR $(600 \mathrm{MHz}$, $\left.\mathrm{CDCl}_{3}\right) \delta 3.73(\mathrm{~d}, J=14 \mathrm{~Hz}, 4 \mathrm{H}), 3.87(\mathrm{~s}, 3 \mathrm{H}), 3.87(\mathrm{~s}, 3 \mathrm{H}), 7.03(\mathrm{~d}, J=2 \mathrm{~Hz}, 2 \mathrm{H})$, $7.13(\mathrm{t}, J=4 \mathrm{~Hz}, 1 \mathrm{H}), 7.69(\mathrm{~d}, J=2 \mathrm{~Hz}, 2 \mathrm{H}), 8.26(\mathrm{t}, J=7 \mathrm{~Hz}, 2 \mathrm{H}) ;{ }^{13} \mathrm{C}$ NMR $(100$ $\left.\mathrm{MHz}, \mathrm{DMSO}-d_{6}\right) \delta 55.98,60.88,114.91,120.76,123.87,129.57,146.49,152.50,165.65$; IR (solid) 3347, 3003, 2942, 2835, 1638, 1575, 1507, 1469, 1426, 1315, 1259, 1072, 991, $912 \mathrm{~cm}^{-1}$; HRMS-FAB (m/z): [M] ${ }^{+}$calcd 388.1644; found 388.1632; m. p. $85-87^{\circ} \mathrm{C}$.

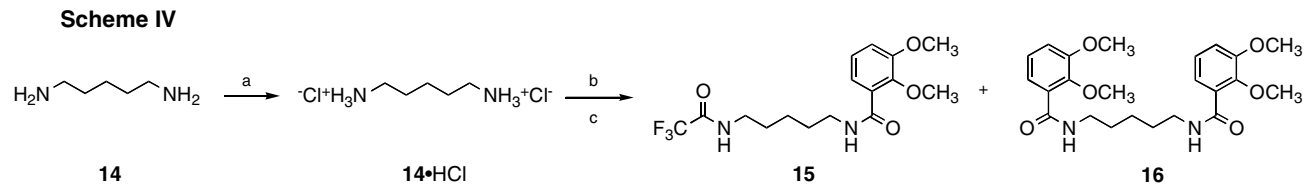

a) 2 eq anhydrous $\mathrm{HCl}$; b) 1 eq $t$ - $\mathrm{BuOK} / \mathrm{CH}_{2} \mathrm{Cl}_{2} ; 1$ eq $\mathrm{CF}_{3} \mathrm{CO}_{2} \mathrm{Et} / \mathrm{CH}_{2} \mathrm{Cl}_{2} ;$ c) 2 eq $t$-BuOK/CH2Cl${ }_{2} ; 1.01$ eq 2,3-dimethoxybenzoyl chloride/CH $\mathrm{Cl}_{2}$

$N$-(2,3-dimethoxybenzoyl)- $N$ '-trifluoroacetyl-1,5-diaminopentane (15): A solution of $0.200 \mathrm{~g}$ (1.14 mmol) 1,5-diaminopentane dihydrochloride $\mathbf{1 4} \mathbf{2} \mathbf{H C l}$ in $80 \mathrm{~mL}$ of anhydrous methanol was reacted with $1.142 \mathrm{~mL}$ of $1.0 \mathrm{M} t$-BuOK solution diluted in 5 $\mathrm{mL} \mathrm{CH}_{2} \mathrm{Cl}_{2}$, added dropwise via syringe pump over $1 \mathrm{~h}$ at $0{ }^{\circ} \mathrm{C}$. After stirring the mixture for an additional $3 \mathrm{~h}$ at room temperature, a solution of $0.136 \mathrm{~mL}$ of ethyl trifluoroacetate $\left(1.14 \mathrm{mmol}\right.$ ) in $10 \mathrm{~mL} \mathrm{CH} \mathrm{Cl}_{2}$ was added dropwise to the mixture within $1 \mathrm{~h}$. Upon stirring overnight, $2.284 \mathrm{~mL}(2.28 \mathrm{mmol})$ of $1.0 \mathrm{M} t$-BuOK solution was added to it within $5 \mathrm{~min}$ and after an additional half an hour, $0.231 \mathrm{~g}(1.15 \mathrm{mmol})$ of 2,3dimethoxybenzoyl chloride dissolved in $5 \mathrm{~mL} \mathrm{CH}_{2} \mathrm{Cl}_{2}$ was added to it and stirred for 30 min. The solvent was removed by rotary evaporation; the residue suspended in ethyl acetate and gravity filtered. Concentration of the filtrate afforded $0.406 \mathrm{~g} \mathrm{(98 \% )}$ crude product as orange oil. Column chromatography of the crude, using gradient mixtures of EtOAc/Hexanes, afforded $0.343 \mathrm{~g} 15(83 \%)$ and $0.022 \mathrm{~g} \mathbf{1 6}(9 \%)$. Characterization: ${ }^{1} \mathrm{H}$ NMR $\left(400 \mathrm{MHz}, \mathrm{CDCl}_{3}\right) \delta 1.33-1.81(\mathrm{~m}, 6 \mathrm{H}), 3.45(\mathrm{dd}, J=10 \mathrm{~Hz}, J=7 \mathrm{~Hz}, 2 \mathrm{H}), 3.54$ $(\mathrm{dd}, J=10 \mathrm{~Hz}, J=6 \mathrm{~Hz}, 2 \mathrm{H}), 4.01(\mathrm{~s}, 3 \mathrm{H}), 4.05(\mathrm{~s}, 3 \mathrm{H}), 6.77(\mathrm{br}, \mathrm{m}, 1 \mathrm{H}), 7.11\left(\mathrm{dd},{ }^{3} J\right.$ $\left.=4 \mathrm{~Hz},{ }^{4} J=1 \mathrm{~Hz}, 1 \mathrm{H}\right), 7.23\left(\mathrm{dd},{ }^{3} J=4 \mathrm{~Hz},{ }^{4} J=1 \mathrm{~Hz}, 1 \mathrm{H}\right), 7.75\left(\mathrm{dd},{ }^{3} J=4 \mathrm{~Hz},{ }^{4} J=1\right.$ $\mathrm{Hz}, 1 \mathrm{H}), 8.11$ (br, m, $1 \mathrm{H}) ;{ }^{13} \mathrm{C} \mathrm{NMR}\left(75 \mathrm{MHz}, \mathrm{CDCl}_{3}\right) \delta 23.82,28.09,29.25,55.98$, $56.08,61.15,62.07,113.95,115.32,117.30,117.77,121.58,122.48,123.58,124.33$, $124.79,126.46,147.30,148.11,152.06,152.46,156.61,157.10,157.59,158.08,165.42$; IR (film) 3362, 3271, 3078, 2932, 1712, 1641, 1535, 1469, 1307, 1264, 1206, 1178, 1153, 1075, 994, 809, 751 $\mathrm{cm}^{-1}$; HRMS-FAB $(\mathrm{m} / \mathrm{z}):[\mathrm{M}+\mathrm{H}]^{+}$calcd 363.1536; found 363.1532 .

$\boldsymbol{N}$-(2,3-dimethoxybenzoyl)- $\boldsymbol{N}$ '-trifluoroacetyl-1,5-diaminopentane (16): ${ }^{1} \mathrm{H}$ NMR (400 $\left.\mathrm{MHz}, \mathrm{CDCl}_{3}\right) \delta 1.58-1.59(\mathrm{~m}, 2 \mathrm{H}), 1.72-1.77(\mathrm{~m}, 4 \mathrm{H}), 3.54(\mathrm{dd}, J=10 \mathrm{~Hz}, J=6 \mathrm{~Hz}, 4$ H), $3.91(\mathrm{~s}, 6 \mathrm{H}), 3.95(\mathrm{~s}, 6 \mathrm{H}), 7.09$ (dd, $J=4 \mathrm{~Hz}, J=1 \mathrm{~Hz}, 2 \mathrm{H}), 7.19$ (t, $J=8 \mathrm{~Hz}, 2 \mathrm{H})$, $7.73(\mathrm{dd}, J=5 \mathrm{~Hz}, J=2 \mathrm{~Hz}, 2 \mathrm{H}), 8.03$ (br, m, $1 \mathrm{H}) ;{ }^{13} \mathrm{C} \mathrm{NMR}\left(100 \mathrm{MHz}, \mathrm{CDCl}_{3}\right) \delta$ $24.55,29.36,39.15,56.12,61.23,115.41,122.91,124.32,127.09$, 147.55, 152.62, 165.15; IR (film) 3384, 3206, 2960, 1655, 1630, 1578, 1306, 1159, 1074, 1015, $986 \mathrm{~cm}^{-1}$; HRMS-FAB (m/z): $[\mathrm{M}+\mathrm{H}]^{+}$calcd 431.2181; found 431.2182. 


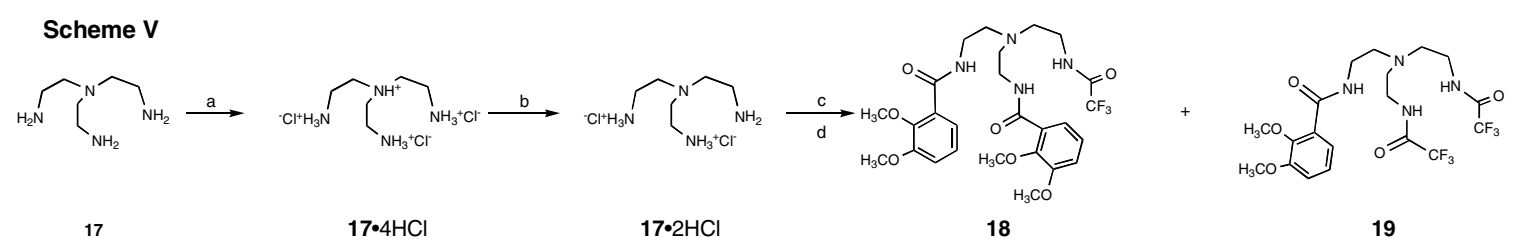

a) anhydrous $\mathrm{HCl}$; b) 2 eq $t$-BuOK/THF; c) 1 eq $\mathrm{CF}_{3} \mathrm{CO}_{2} \mathrm{Et} / \mathrm{THF}$; d) 6 eq $t$-BuOK/THF; e) 2 eq 2,3-dimethoxybenzoyl chloride/THF

2-trifluoroacetyl-2-aminoethyl)-bis(2,3-dimethoxybenzoyl-2-aminoethyl)amine (18): TREN dihydrochloride $0.300 \mathrm{~g}(1.03 \mathrm{mmol})$ was suspended in 100 of a $1: 1$ $\mathrm{DMSO} / \mathrm{MeOH}$ (vol.) solution mixture, heated until complete dissolution, and magnetically stirred for $15 \mathrm{~min}$. To this solution, $t$-BuOK $1.03 \mathrm{~mL}(1.03 \mathrm{mmol})$ dissolved in $10 \mathrm{~mL}$ THF was slowly added via mechanical syringe pump over $1 \mathrm{~h}$ and left stirring for an additional $3 \mathrm{~h}$. Ethyl trifluoroacetate $0.122 \mathrm{~mL}(1.03 \mathrm{mmol})$, dissolved in $10 \mathrm{~mL}$ THF, was slowly added via mechanical syringe pump and left stirring overnight. The resulting solution was reacted with $t$-BuOK, $6.18 \mathrm{~mL}(6.18 \mathrm{mmol})$ followed by 2,3dimethoxybenzoyl chloride, $0.212 \mathrm{~g}(2.06 \mathrm{mmol})$, dissolved in $5 \mathrm{~mL}$ THF. The resulting mixture was stirred overnight. The crude product was extracted with $\mathrm{CHCl}_{3}(3 \times 50 \mathrm{~mL})$, washed with distilled water, brine, dried over $\mathrm{MgSO}_{4}$, and concentrated to afford $0.510 \mathrm{~g}$ (87\%) of crude product. Column chromatography with gradient mixtures of EtOAc (30$80 \%$ ) and hexanes eluted unsymmetrical 18 and 19 in $75 \%$ and $6 \%$ yields, respectively. (18): ${ }^{1} \mathrm{H} \mathrm{NMR}\left(400 \mathrm{MHz}, \mathrm{CDCl}_{3}\right) 2.81(\mathrm{~m}, 6 \mathrm{H}), 3.43(\mathrm{~d}, J=7.2 \mathrm{~Hz}, 2 \mathrm{H}), 3.50$ (dd, $J=$ $12.8 \mathrm{~Hz}, J=6.8 \mathrm{~Hz}, 4 \mathrm{H}), 3.81(\mathrm{~s}, 6 \mathrm{H}), 3.82(\mathrm{~s}, 6 \mathrm{H}), 6.96(\mathrm{dd}, J=8.6 \mathrm{~Hz}, J=1.6 \mathrm{~Hz}, 2$ H, 7.02 (t, $J=8.0 \mathrm{~Hz}, 2 \mathrm{H}), 7.48$ (d, $J=7.2 \mathrm{~Hz}, 2 \mathrm{H}), 8.07$ (br, s, $1 \mathrm{H}), 8.21$, (bs, $2 \mathrm{H})$; ${ }^{13} \mathrm{C}$ NMR $\left(100 \mathrm{MHz}, \mathrm{CDCl}_{3}\right) \delta 23.78,24.55,28.13,28.36,29.37,29.65,38.91,39.53$, $39.87,44.36,46.02,56.13,61.23,115.41,115.54,122.92,124.32,126.81,127.10$, 128.80, 130.80, 147.55, 152.62, 165.14, 165.52; IR (neat): 3367, 3261, 3073, 2942, 2835, 1714, 1641, 1575, 1527, 1472, 1459, 1426, 1307, 1262, 1204, 1181, 1153, 1070, 991, 910, 840, 754, $723 \mathrm{~cm}^{-1}$; HRMS-FAB (m/z): [M] ${ }^{+}$calcd 570.2301; found 570.2315.

\section{N-(2,3-dimethoxybenzoyl-2-aminoethyl)-N',N'-bis(trifluoroacetyl-2-}

aminoethyl)amine (19): ${ }^{1} \mathrm{H} \mathrm{NMR}\left(400 \mathrm{MHz} \mathrm{CDCl}_{3}\right) 2.74(\mathrm{~m}, 6 \mathrm{H}), 3.40(\mathrm{dd}, J=12 \mathrm{~Hz}$, $J=6 \mathrm{~Hz}, 4 \mathrm{H}), 3.54(\mathrm{dd}, J=9.6 \mathrm{~Hz}, J=4 \mathrm{~Hz}, 2 \mathrm{H}$ ), 3.90 (s, $3 \mathrm{H}), 3.92$ (s, $3 \mathrm{H}), 7.07$ (dd, $J=8.8 \mathrm{~Hz}, J=2 \mathrm{~Hz}, 1 \mathrm{H}), 7.16(\mathrm{t}, J=8 \mathrm{~Hz}, 1 \mathrm{H}), 7.69(\mathrm{~d}, J=6.8 \mathrm{~Hz}, 1 \mathrm{H}), 7.85(\mathrm{br}, \mathrm{s}, 2$ $\mathrm{H}), 8.46(\mathrm{t}, J=7.2 \mathrm{~Hz}, 1 \mathrm{H}) ;{ }^{13} \mathrm{C}$ NMR $\left(100 \mathrm{MHz}, \mathrm{CDCl}_{3}\right) \delta 23.78,24.55,28.13,28.38$, $29.37,29.65,38.91,39.53,39.87,44.36,46.02,56.13,61.23,115.41,115.54,122.92$, $124.32,126.81,127.10,128.80,130.80,147.55,152.61,165.14,165.51$; IR (neat): 3347 , 3266, 3084, 2841, 1709, 1638, 1575, 1527, 1474, 1459, 1426, 1307, 1259, 1211, 1183 , 1153, 1070, 989, 910, 842, $723 \mathrm{~cm}^{-1}$; HRMS-FAB (m/z): $[\mathrm{M}]^{+}$calcd 503.172; found 503.5737 .

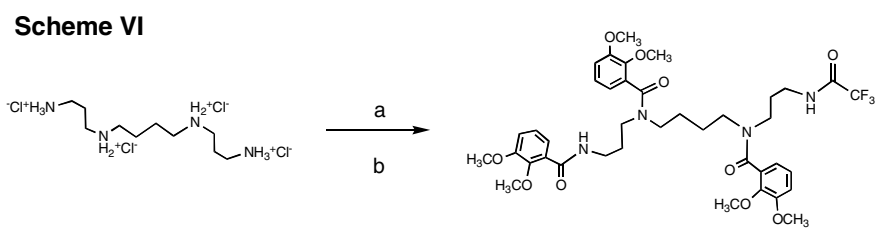

$20 \cdot 4 \mathrm{HCl}$

21

a) 1 eq $t$-BuOK; b) 1 eq $\mathrm{CF}_{3} \mathrm{CO}_{2} \mathrm{Et}$; c) 6 eq $t$-BuOK; 3.1 eq 2,3-dimethoxybenzoyl chloride 
$\mathrm{N}^{1}$-Trifluoroacetyl-( $\mathrm{N}^{4}, \mathrm{~N}^{9}, \mathrm{~N}^{12}$-Tri-2,3-dimethoxybenzoyl)-1,12-diamino-4,9-

diazadodecane (21): A solution of $1.400 \mathrm{~mL} 1.01 \mathrm{M} t$-BuOK (1.410 mmol) in THF was diluted with $10 \mathrm{~mL} \mathrm{CH} \mathrm{Cl}_{2}$ and added dropwise over $1 \mathrm{~h}$ (syringe pump) to a second solution of $0.500 \mathrm{~g}(1.410 \mathrm{mmol})$ spermine tetrahydrochloride $\mathbf{2 0} \cdot \mathbf{4 H C l}$ in $250 \mathrm{~mL}$ methanol. After $3 \mathrm{~h}$, the reaction mixture was cooled down in an ice bath and ethyl trifluoroacetate $(0.171 \mathrm{~mL}, 1.410 \mathrm{mmol})$ in $20 \mathrm{~mL} \mathrm{CH}_{2} \mathrm{Cl}_{2}$ was added via syringe pump. The mixture was allowed to warm to room temperature, stirred for $3 \mathrm{~h}$, and reacted with $8.400 \mathrm{~mL} 1.01 \mathrm{M} t$-BuOK $(8.400 \mathrm{mmol})$. The mixture was stirred for an additional 10 min before treating it with a solution of 2,3-dimethoxybenzoyl chloride $(0.868 \mathrm{~g}, 4.300$ mmol) in $20 \mathrm{~mL} \mathrm{CH}_{2} \mathrm{Cl}_{2}$. The mixture was stirred for $4 \mathrm{~h}$ and concentrated. The crude product was suspended in $20 \mathrm{~mL}$ EtOAc, gravity filtered, and concentrated. The product was further purified by column chromatography with gradient mixtures of hexanes/EtOAc (30-75\%) to afford $0.960 \mathrm{~g}(87 \%):{ }^{1} \mathrm{H}$ NMR $\left(600 \mathrm{MHz}, \mathrm{CDCl}_{3}\right) \delta 1.13-$ $1.93(\mathrm{~m}, 8 \mathrm{H}), 2.91-3.94(\mathrm{~m}, 26 \mathrm{H}), 6.68-7.10(\mathrm{~m}, 7 \mathrm{H}), 7.63(\mathrm{dt}, J=7 \mathrm{~Hz}, J=1 \mathrm{~Hz}, 1 \mathrm{H})$, $8.35(\mathrm{~m}, 1 \mathrm{H}) ;{ }^{13} \mathrm{C}$ NMR $\left(100 \mathrm{MHz}, \mathrm{CDCl}_{3}\right) \delta 29.31,30.31,31.39,37.97,39.14,39.25$, 43.04 , 44.06, 44.21, 44.40, 45.79, 46.26, 46.58, 49.00, 50.48, 58.32, 64.06, 115.59, $116.04,118.07,120.98,125.37,126.73\left(\mathrm{q}, J=150 \mathrm{~Hz}, C F_{3}\right), 126.96,129.39,129.98$, $133.75,133.96,134.70,147.39,150.14,150.53,155.39,159.89,160.26,167.93,168.143$, 171.92 (q, $\left.J=15 \mathrm{~Hz}, \mathrm{COCF}_{3}\right), 173.32$; IR $\left(\mathrm{CDCl}_{3}\right.$ film) 3384, 2924, 2852, 1720, 1652, 1574, 1536, 1456, 1260, 1217, 1165, $1065 \mathrm{~cm}^{-1}$; HRMS-FAB $(\mathrm{m} / \mathrm{z}):[\mathrm{M}+\mathrm{H}]^{+}$calcd 791.3478; found 791.3496 .

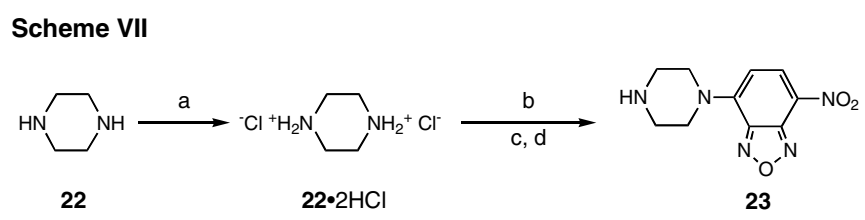

a) 2 eq $\mathrm{HCl}$; b) 1 eq $t$-BuOK; c) NBD chloride; d) $t$-BuOK

Piperazinyl-NBD 23: To a solution of $0.500 \mathrm{~g}$ of piperazine dihydrochoride $(3.100$ $\mathrm{mmol}$ ) in $100 \mathrm{~mL}$ anhydrous DMSO was added dropwise over $1 \mathrm{~h}$ via syringe pump 3.10 $\mathrm{mL}$ of $1.0 \mathrm{M} t$-BuOK solution diluted in $20 \mathrm{~mL} \mathrm{CH} \mathrm{Cl}_{2}$. The resulting mixture was stirred for $2 \mathrm{~h}$. A solution prepared with $0.627 \mathrm{~g} \mathrm{NBD}-\mathrm{Cl}(3.10 \mathrm{mmol})$ in $30 \mathrm{~mL} \mathrm{CH}_{2} \mathrm{Cl}_{2}$ was added dropwise with a mechanical syringe pump, $1 \mathrm{~h}$. After stirring for an additional $2 \mathrm{~h}$, the mixture was treated with $6.20 \mathrm{~mL} 1.0 \mathrm{M} t$-BuOK in THF, stirred for $1 \mathrm{~h}$, extracted with EtOAc $(3 \times 100 \mathrm{~mL})$, washed with $\mathrm{H}_{2} \mathrm{O}$, brine, dried over $\mathrm{MgSO}_{4}$, and concentrated. The resulting red solid was purified by column chromatography with gradient mixtures of $\mathrm{MeOH}(0-50 \%)$ and EtOAc to afford $0.751 \mathrm{~g}(96 \%)$. ${ }^{1} \mathrm{H}$ NMR (300 $\left.\mathrm{MHz}, \mathrm{CDCl}_{3}\right) \delta 3.00(\mathrm{~s}, 1 \mathrm{H}, \mathrm{NH}), 3.10\left(\mathrm{t}, J=5 \mathrm{~Hz}, 4 \mathrm{H}, C_{2}\right), 4.06(\mathrm{t}, J=5 \mathrm{~Hz}), 6.26(\mathrm{~d}, J$ $=9 \mathrm{~Hz}), 8.39(\mathrm{~d}, J=9 \mathrm{~Hz}) ;{ }^{13} \mathrm{C} \mathrm{NMR}\left(100 \mathrm{MHz}, \mathrm{CDCl}_{3}\right) \delta 42.87,45.32,50.74,103.12$, 136.01, 144.63, 145.10; IR (film) 3342, 3099, 2830, 2749, 1603, 1540, 1474, 1426, 1335, $1274,1252,1234,1143,1042,999,910,792,736 \mathrm{~cm}^{-1}$; HRMS-FAB $(\mathrm{m} / \mathrm{z}):[\mathrm{M}+\mathrm{H}]^{+}$ calcd 250.0940; found 250.0929.

\section{Additional Reference}


${ }^{6}$ Lee, G.; Bashara, J. N.; Sabih, G.; Oganesyan, A.; Godjoian, G.; Duong, H.M.; Marinez, E.R.; Gutierrez, C.G. Org. Lett. 2004, 6, 1705-1707.

${ }^{16}$ Perrin, D. D.; Armarego, W. L. F. Purification of Laboratory Chemicals, $3^{\text {rd }}$ Ed.; Pergamon Press, New York, 1988. 

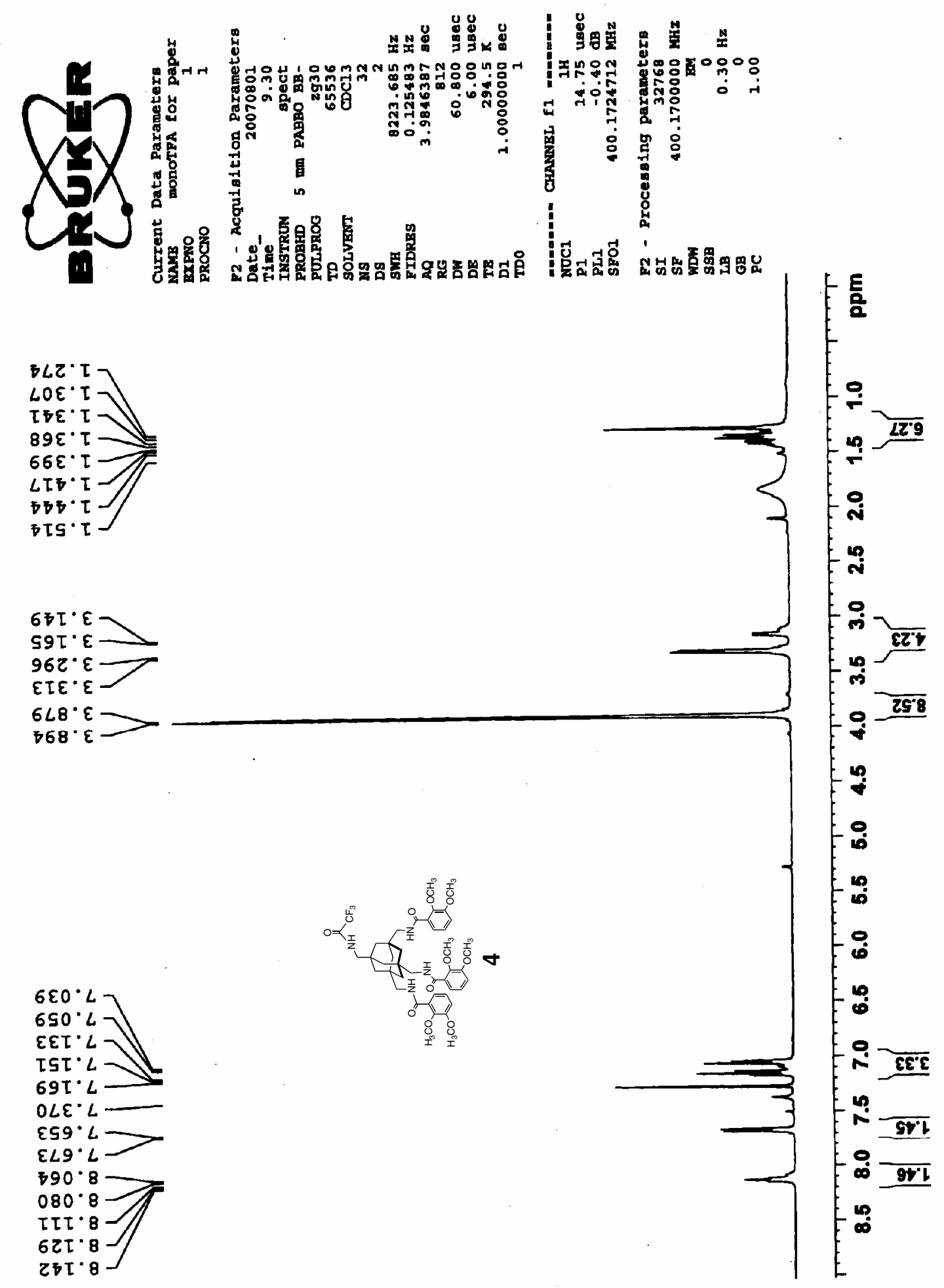

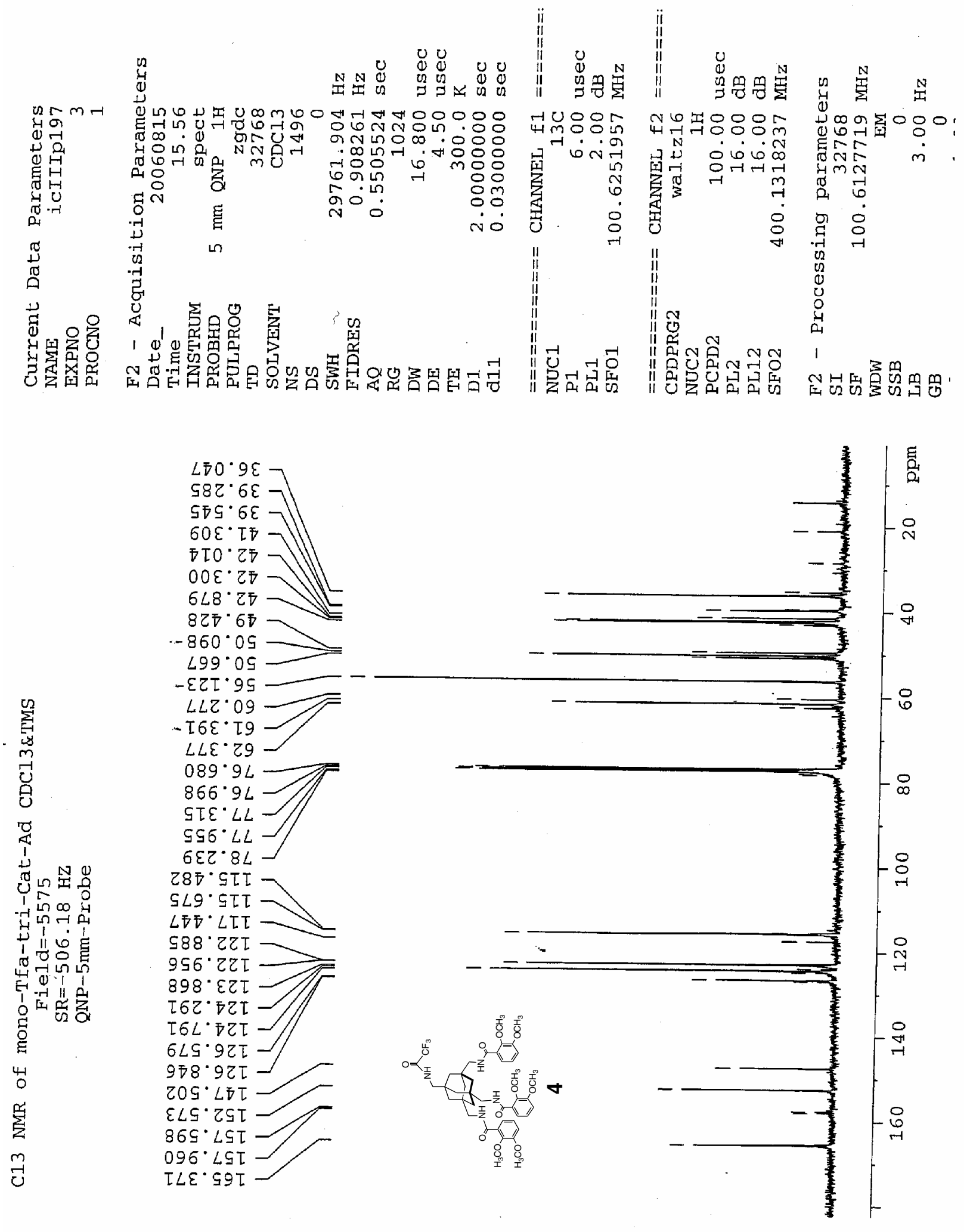


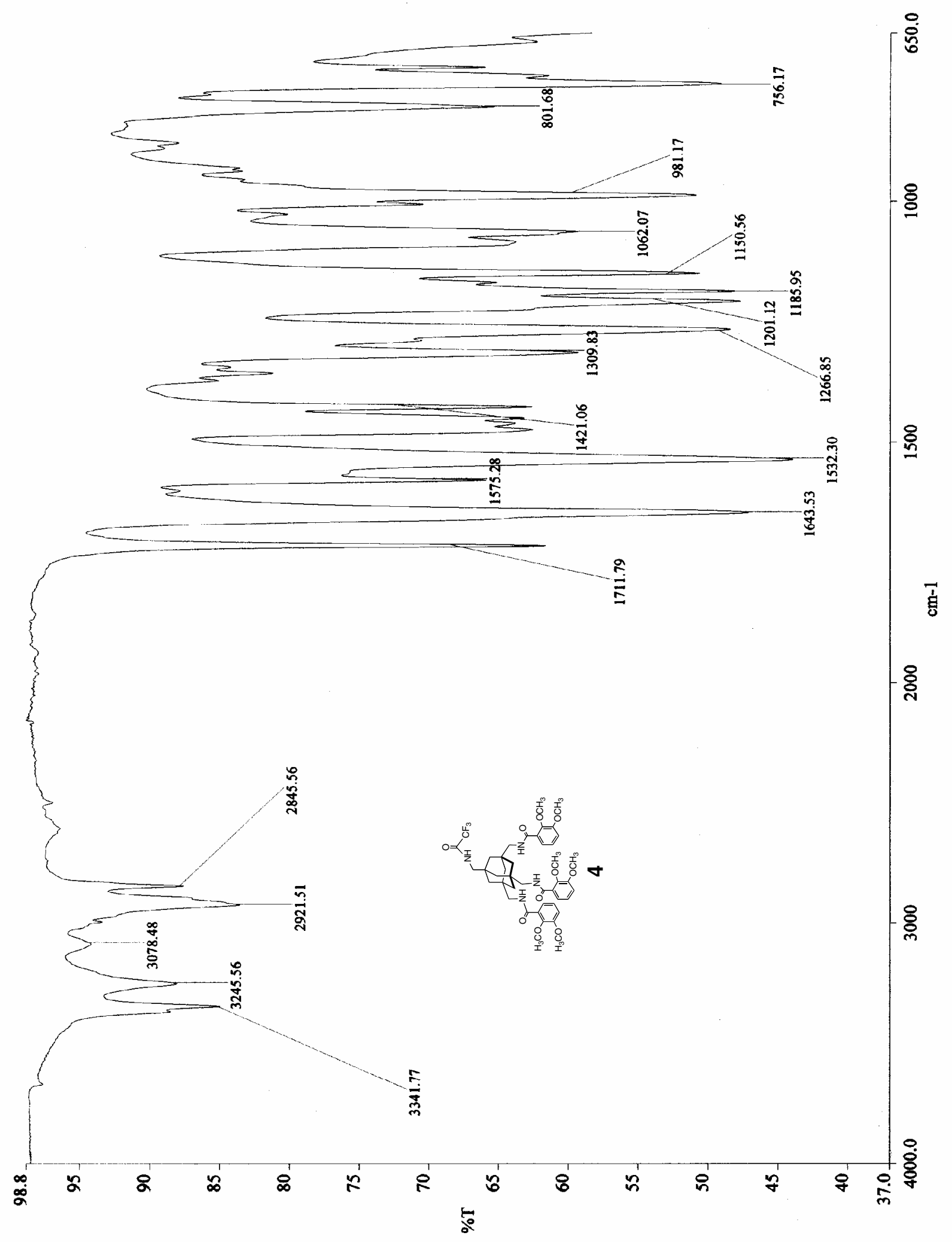

S-11 


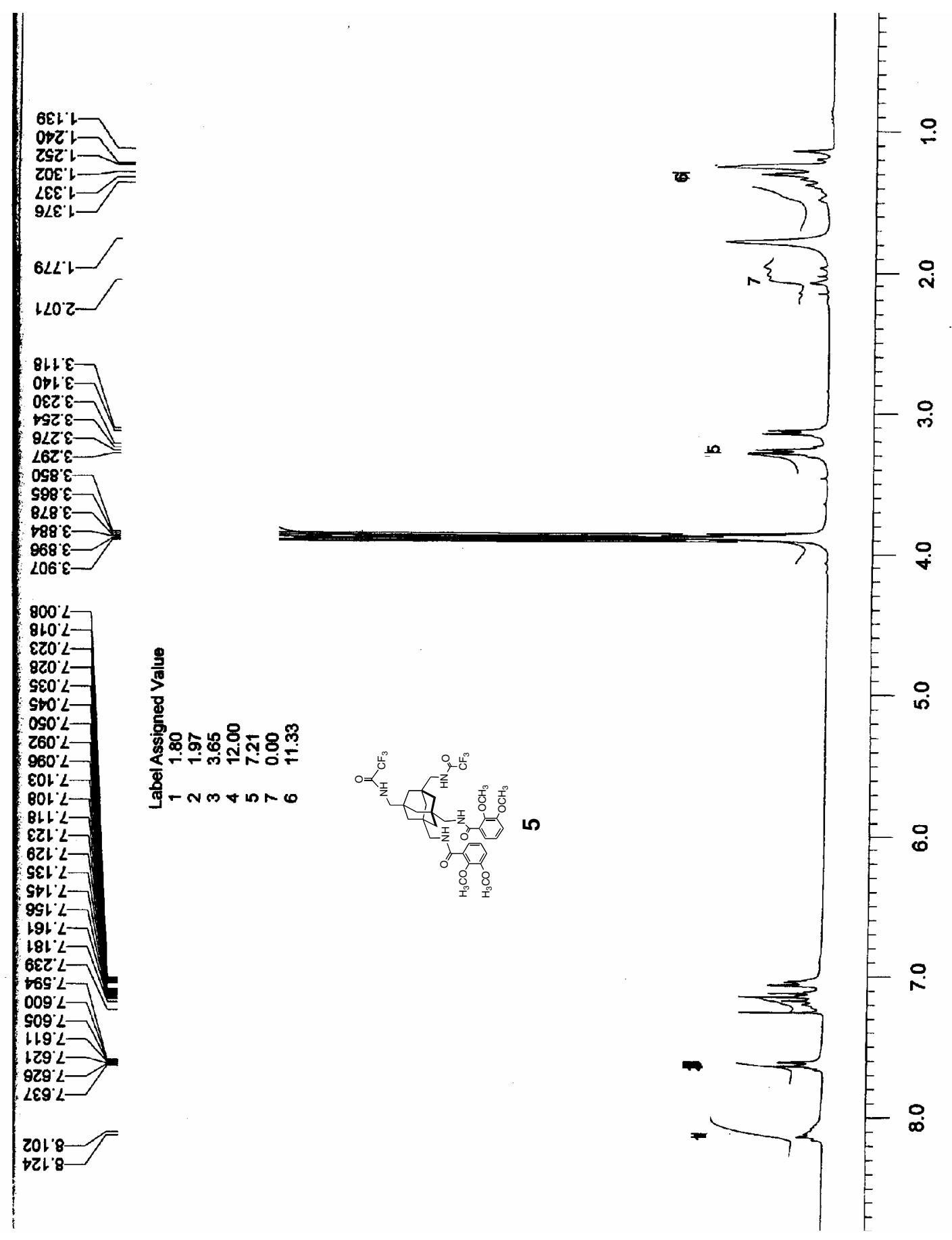




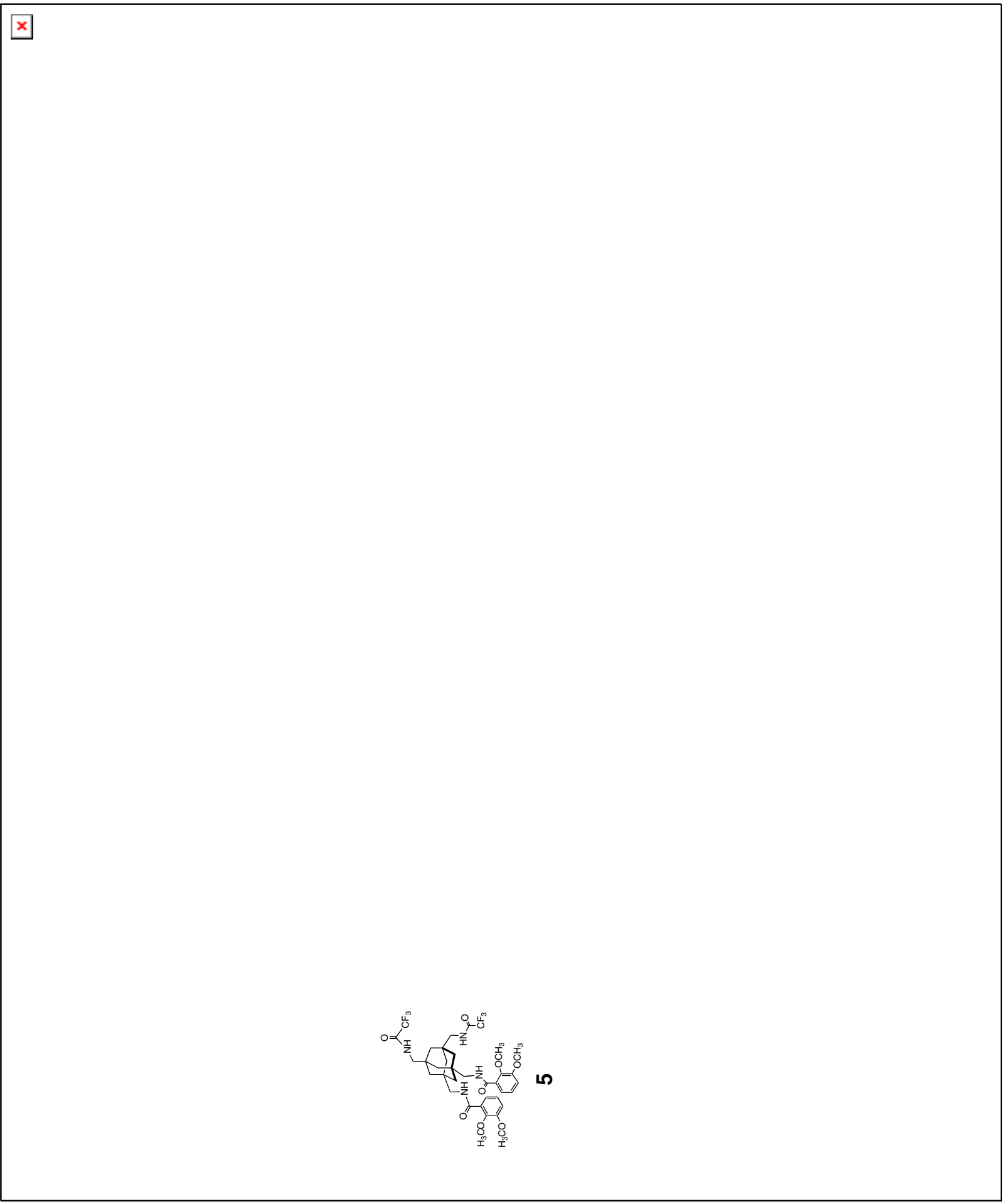

S-13 


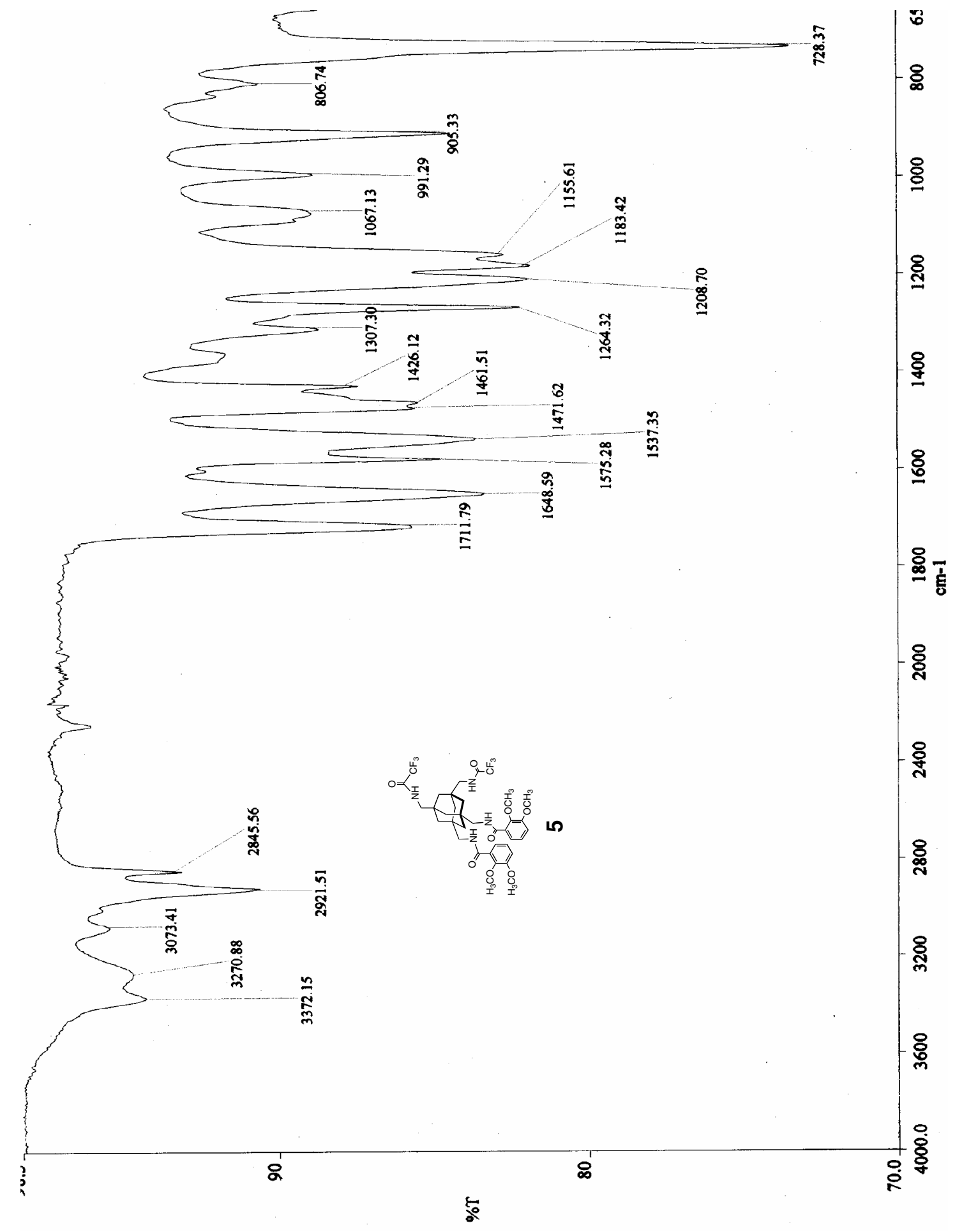




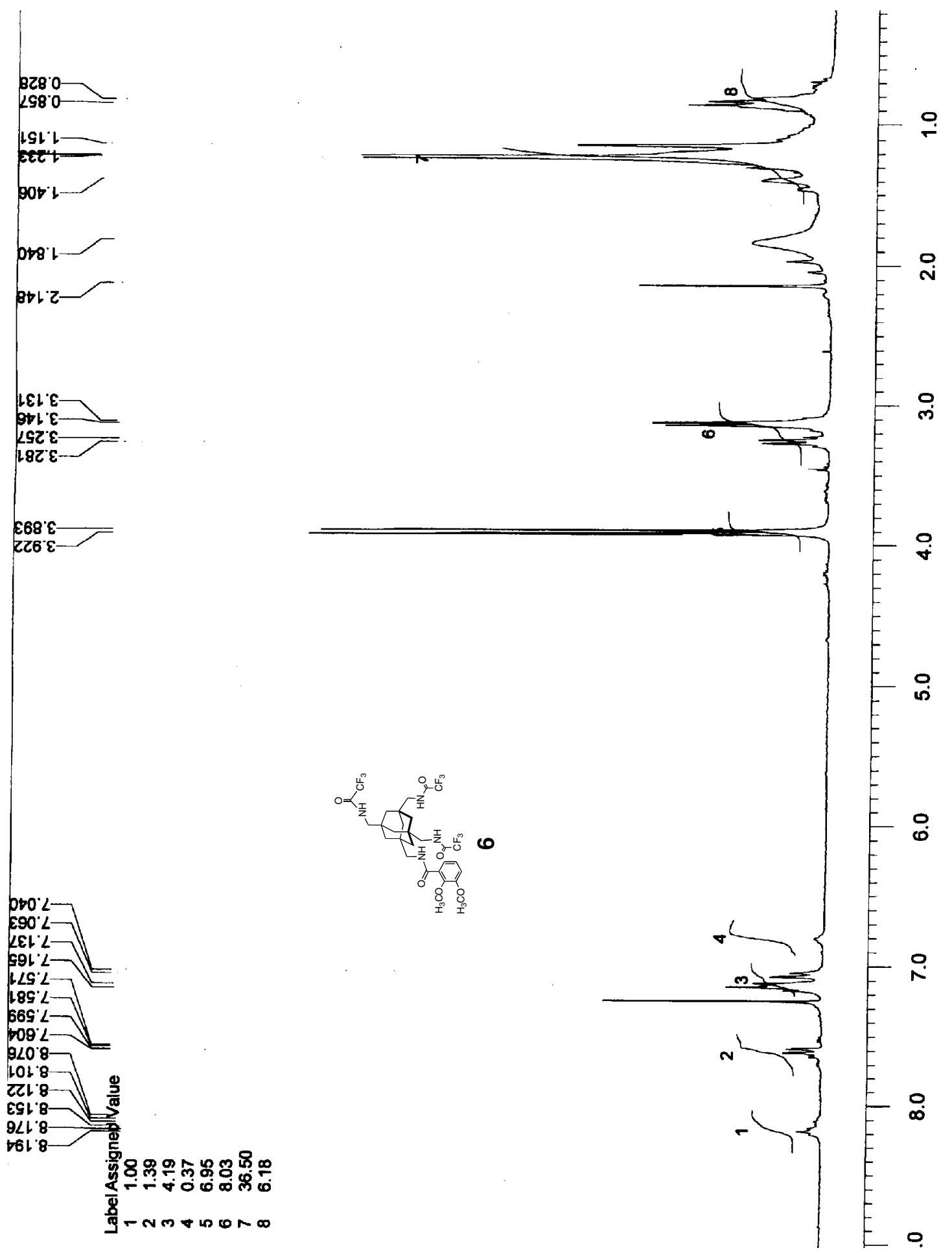




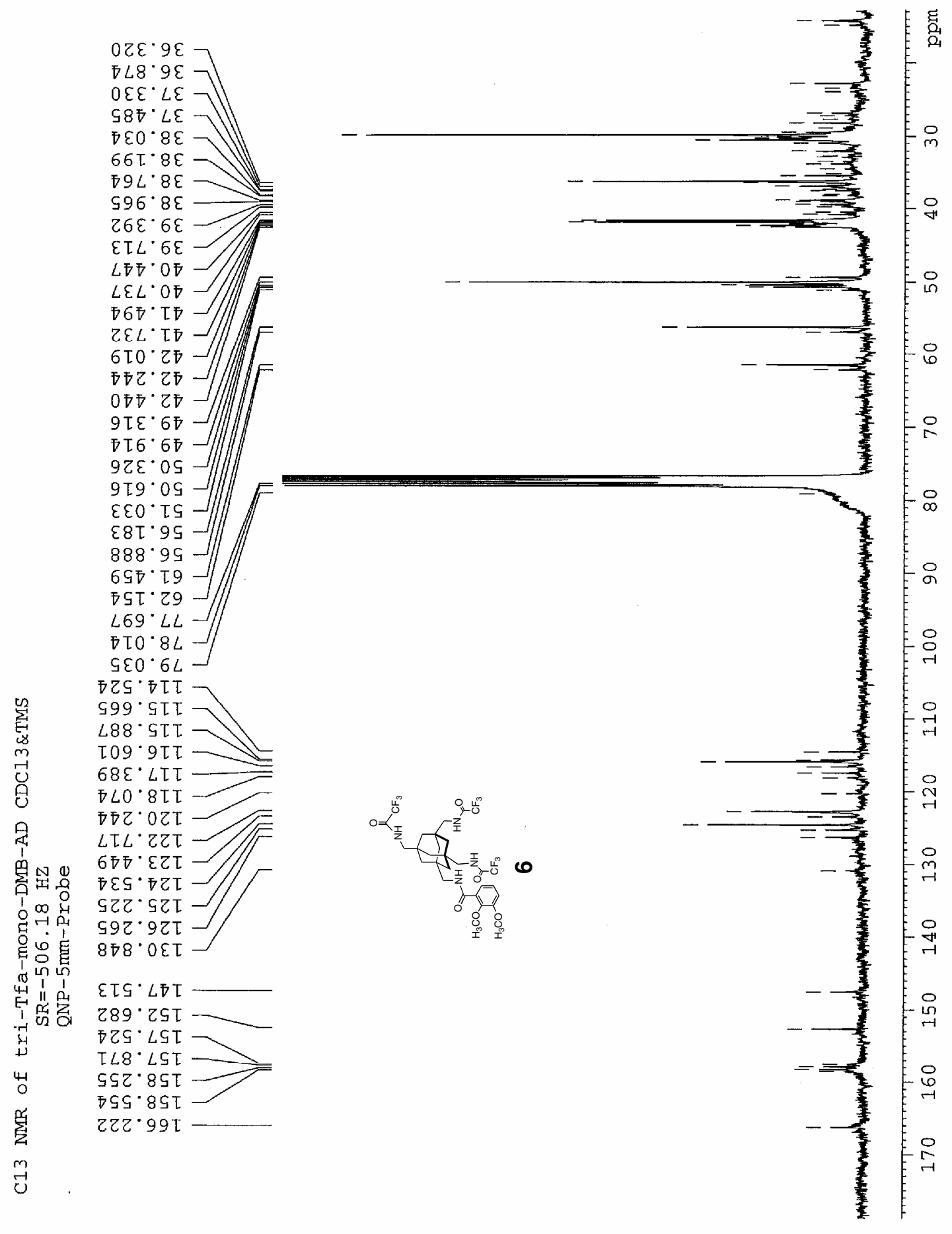




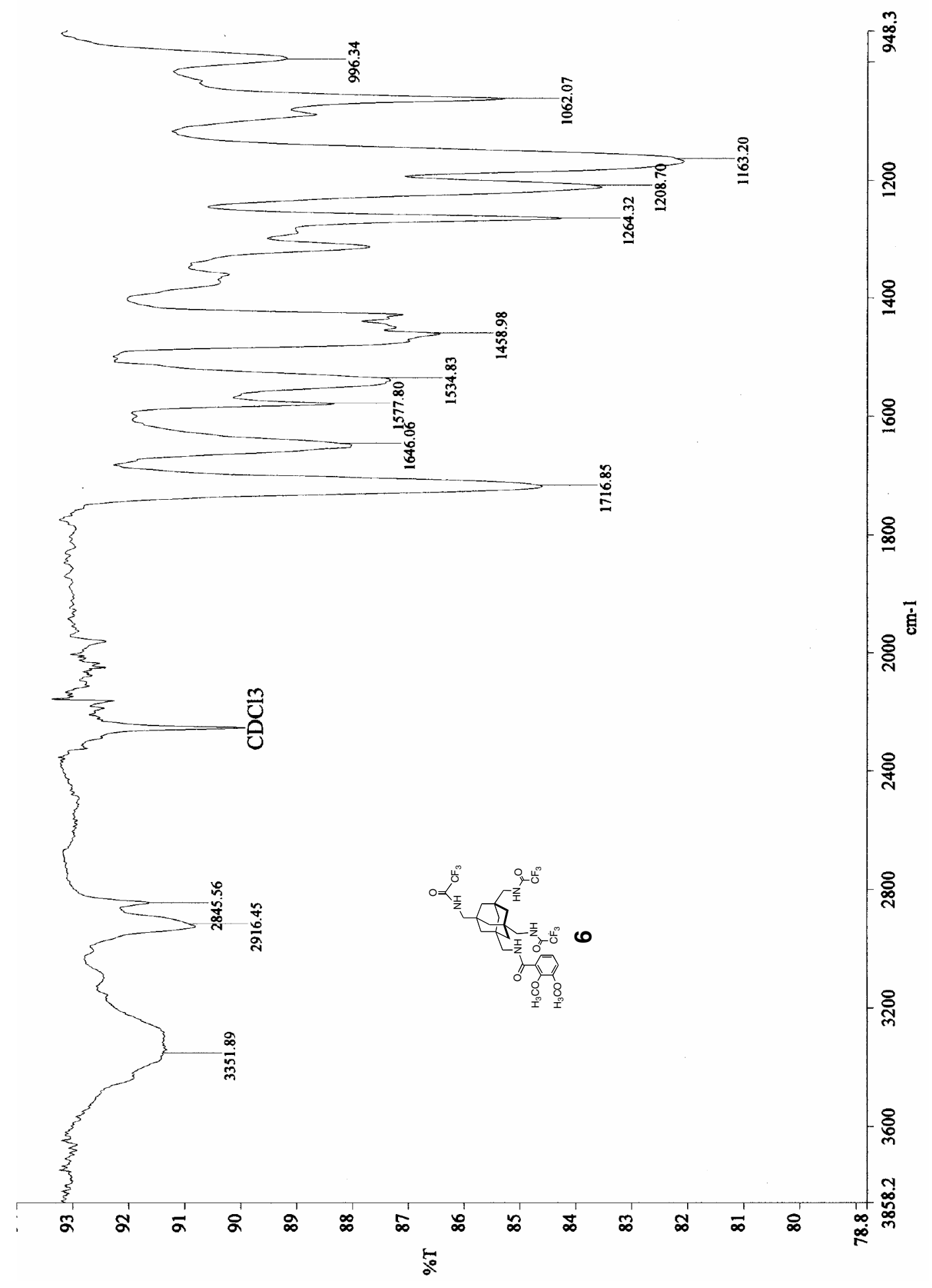




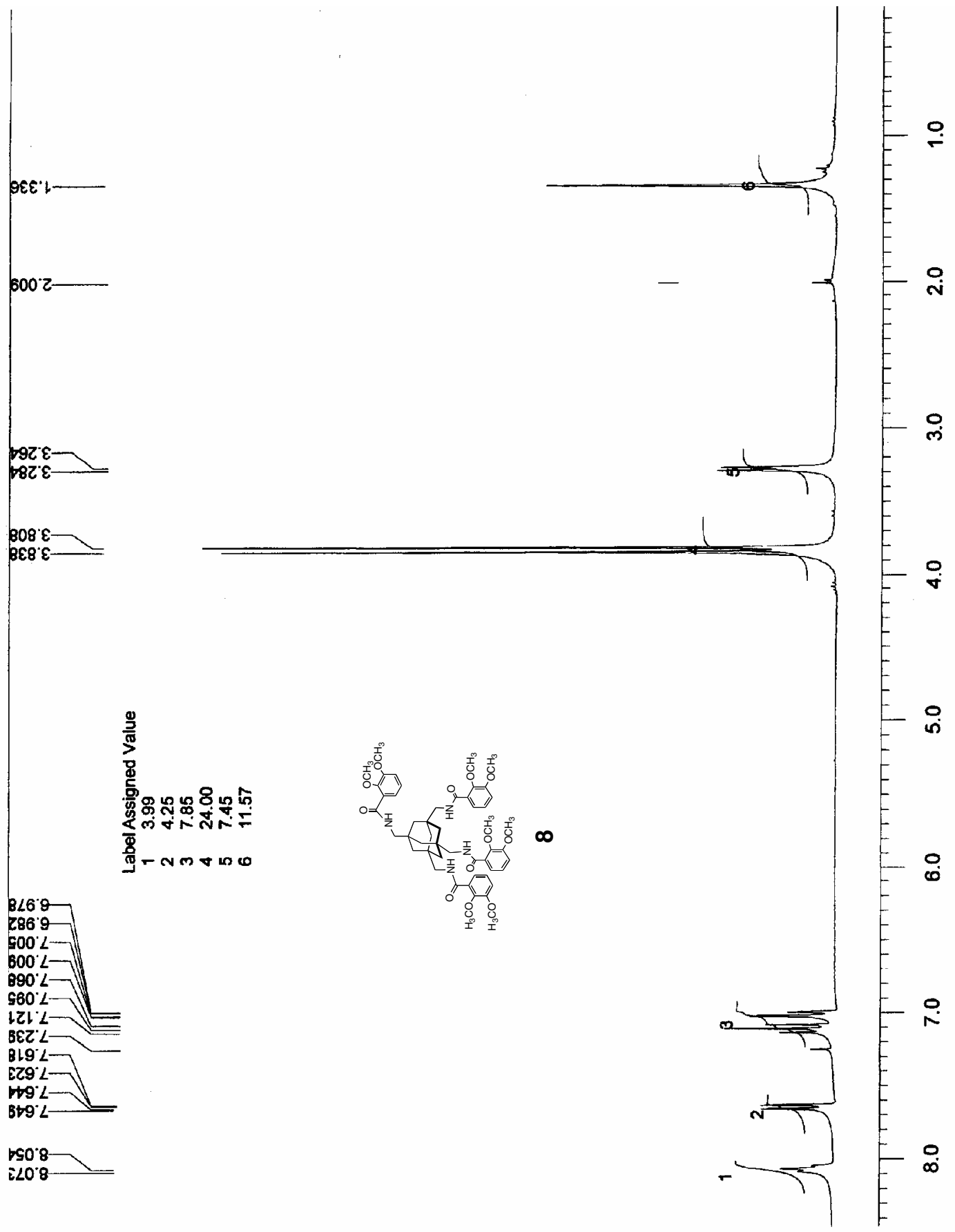




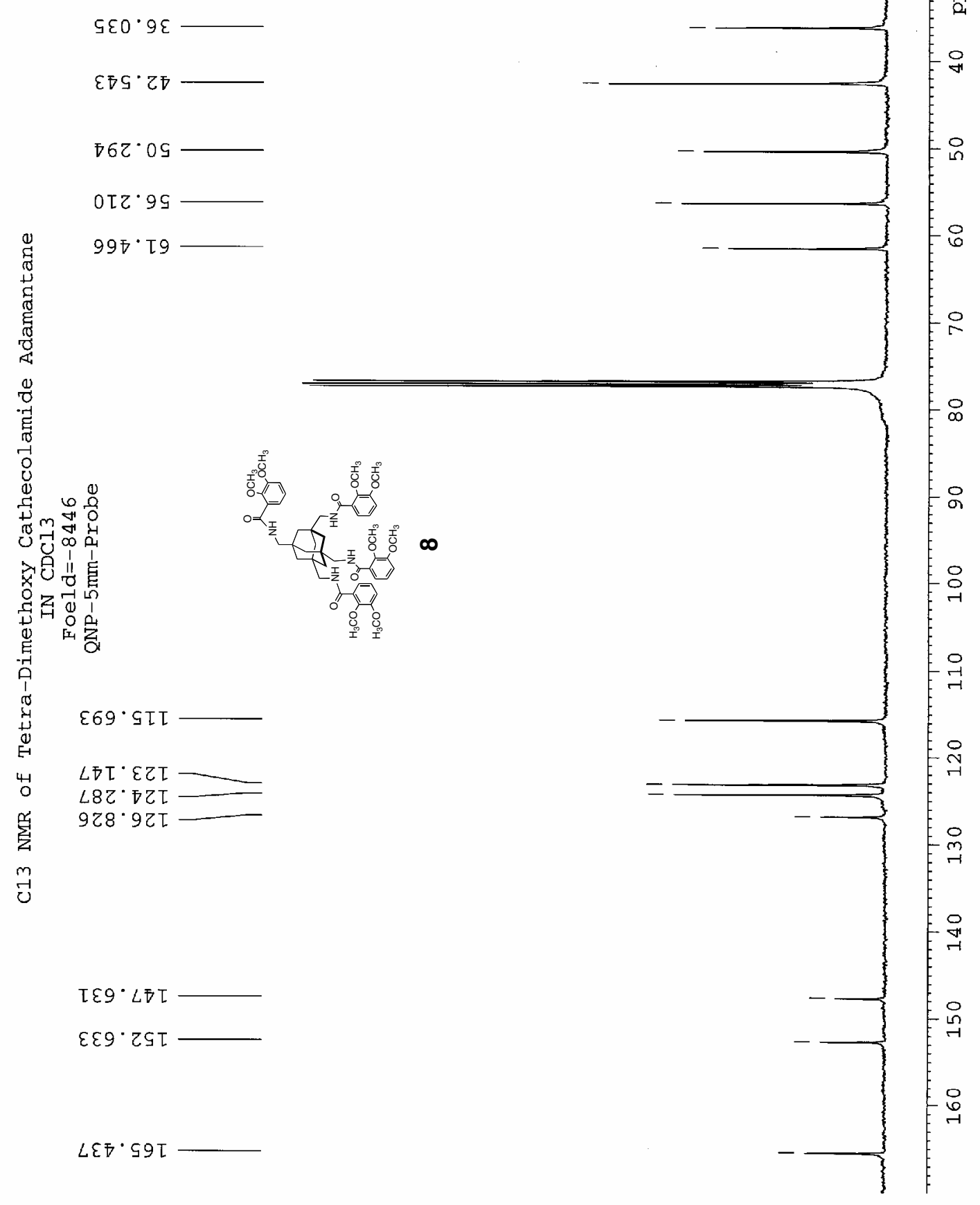




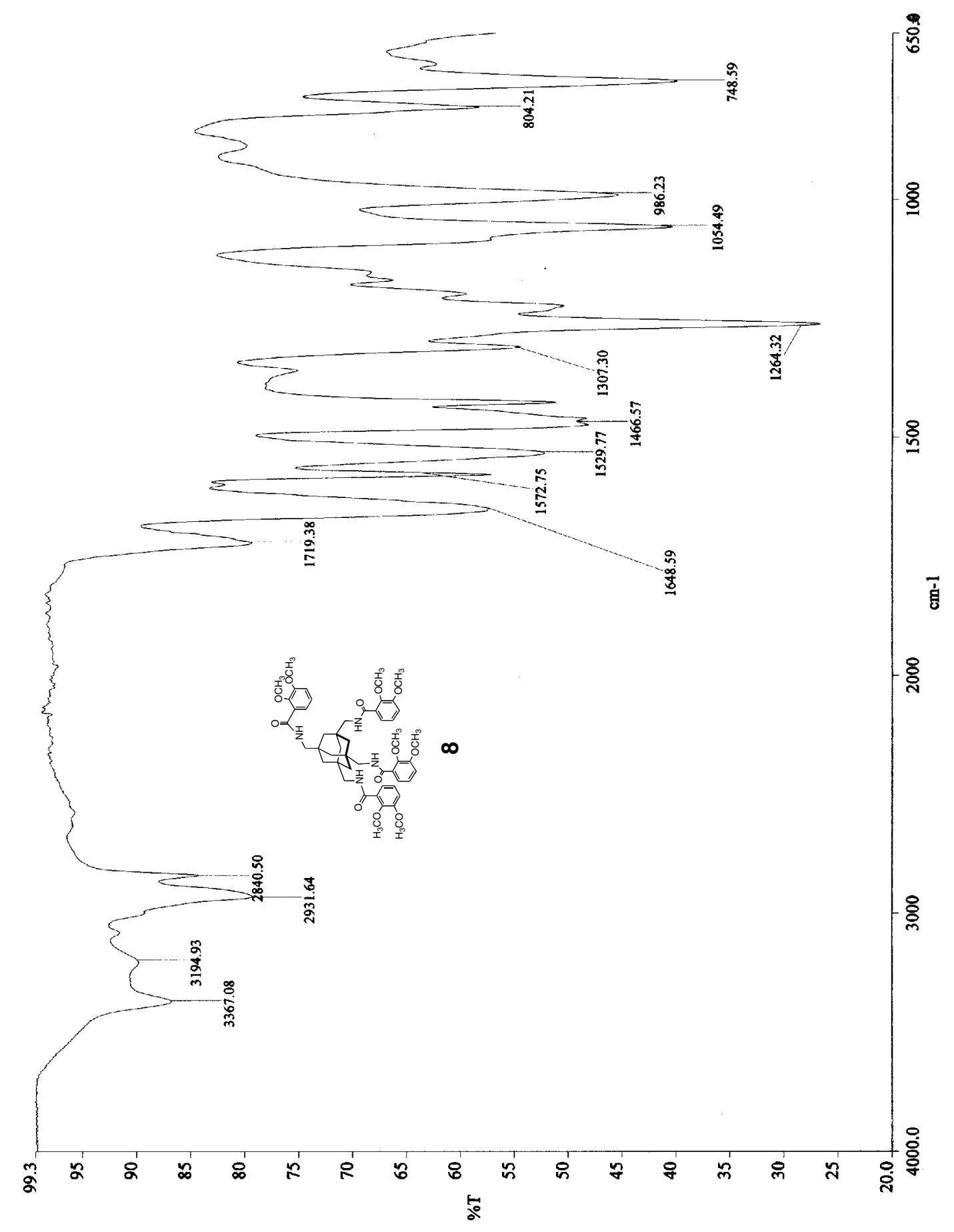


$\angle S 8^{\circ} 0$

$O \nabla Z \cdot I$

๑医

$026^{\circ} \mathrm{L}$

ZII'乙

$80 \varepsilon^{\cdot} \varepsilon$

$068^{\circ} \varepsilon-1$
$668^{\circ}$

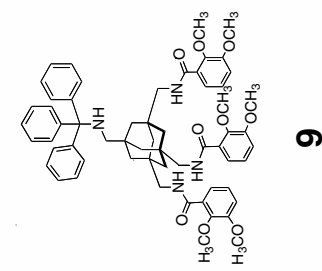

$\overline{D O O} 0^{\circ} \mathrm{L}$

$\angle B T \cdot L$

ISZ $L$

$\varepsilon \nabla \sigma \cdot L-$

$6 \angle 9^{\circ} L$

$\varepsilon 90^{\circ} 8$

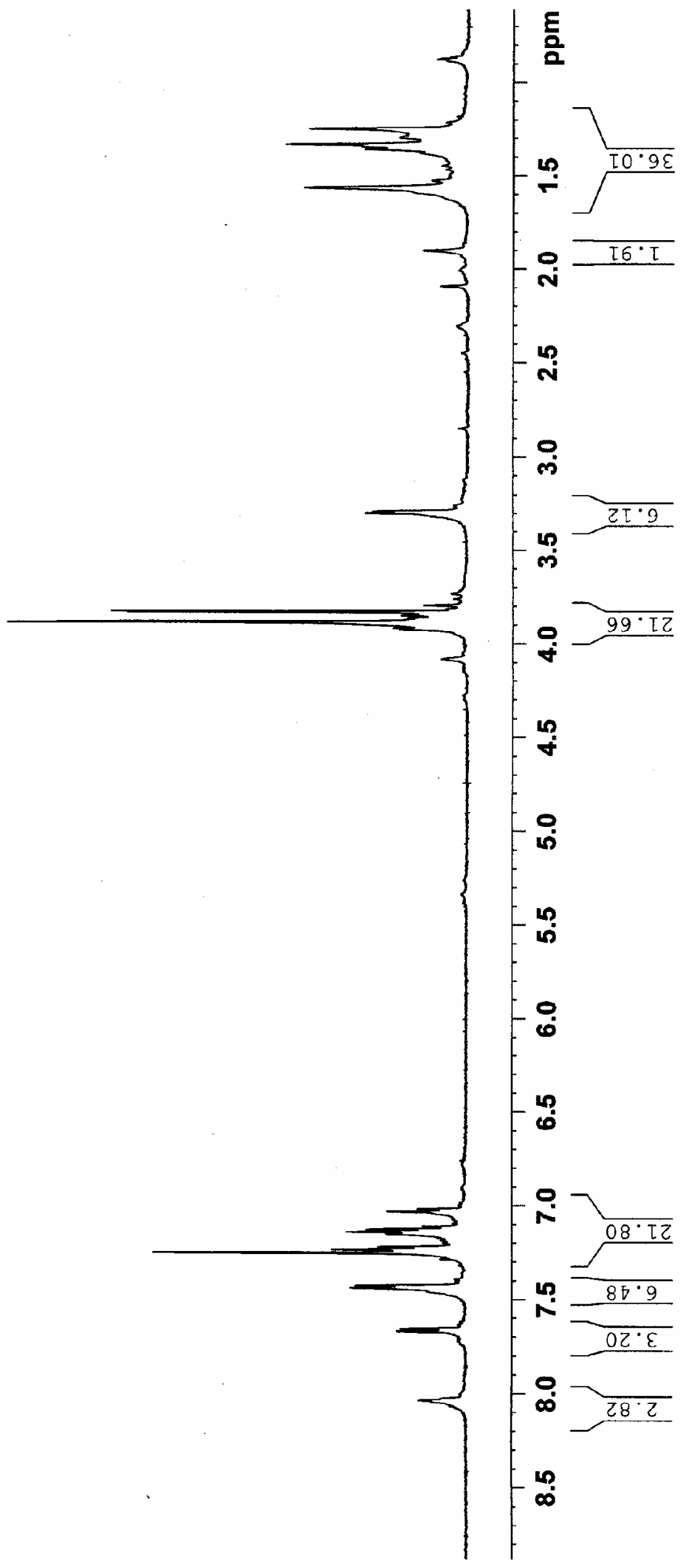




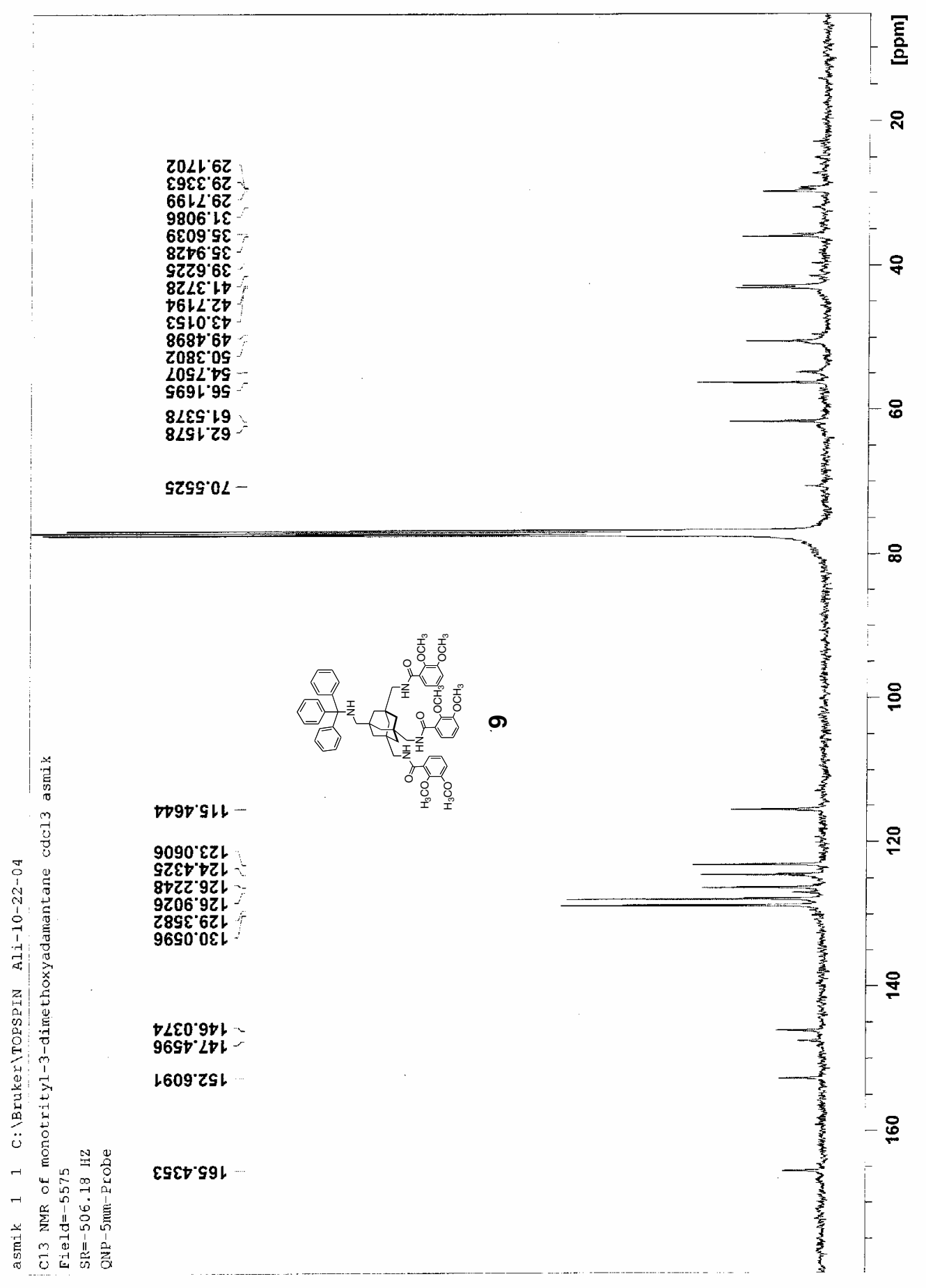




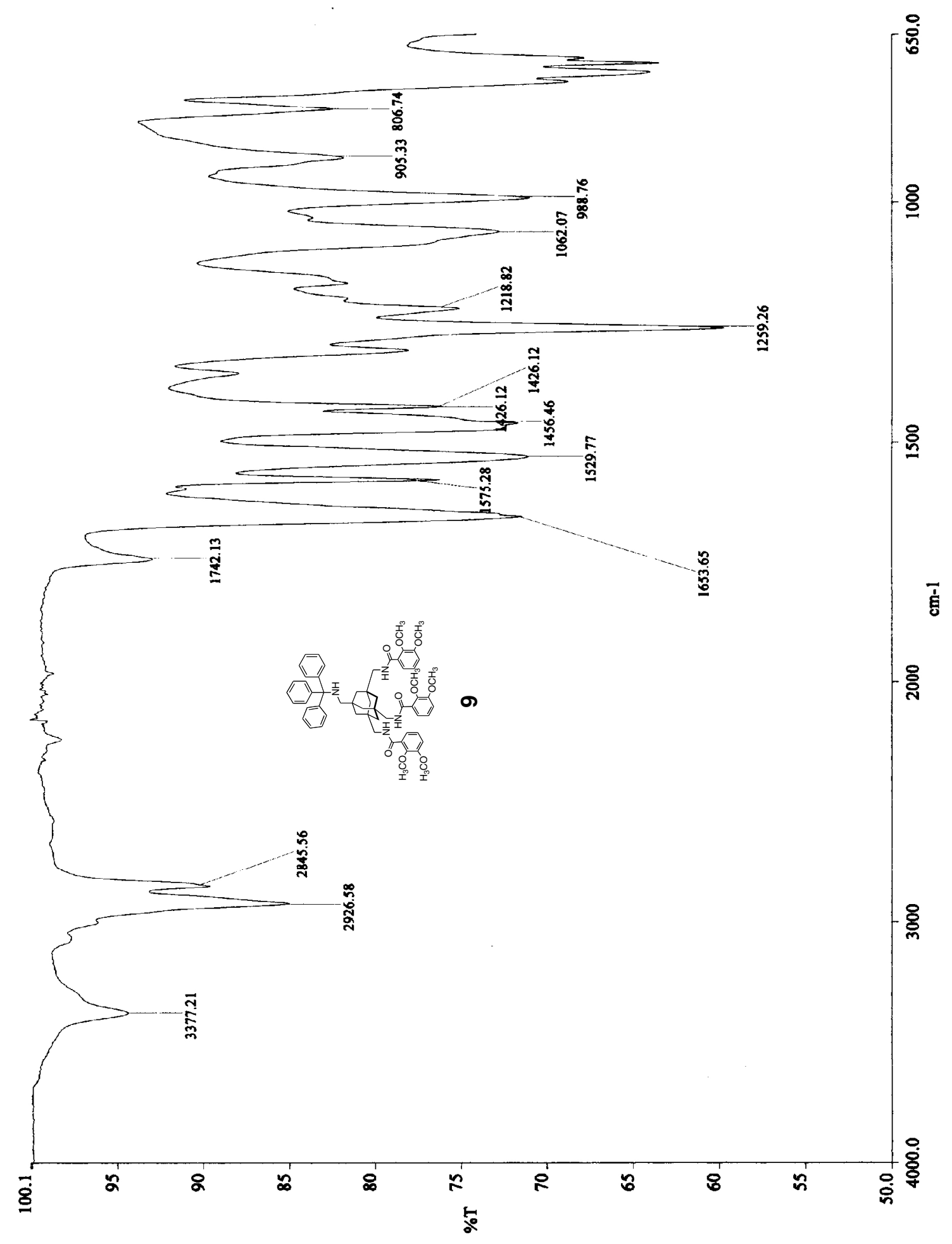




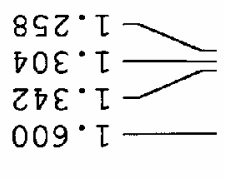

$968^{\circ} \mathrm{I}$

$0 O \varepsilon^{-} \mathcal{E}$

$\angle \varepsilon 8^{\circ} \varepsilon$
$\overline{ } 06^{\circ} \varepsilon$

$806^{\circ} \varepsilon$

$\varepsilon 26^{\circ} \varepsilon$

$S \nabla 0^{\circ} \mathrm{L} \longrightarrow$

$\mathrm{ZST} \cdot \mathrm{L}$

$6 \nabla C^{*} L$

$690^{\circ} L$

$\angle E S^{\circ} L$

$9 \angle 9^{\circ} \mathrm{L}-$

$920 \cdot 8$

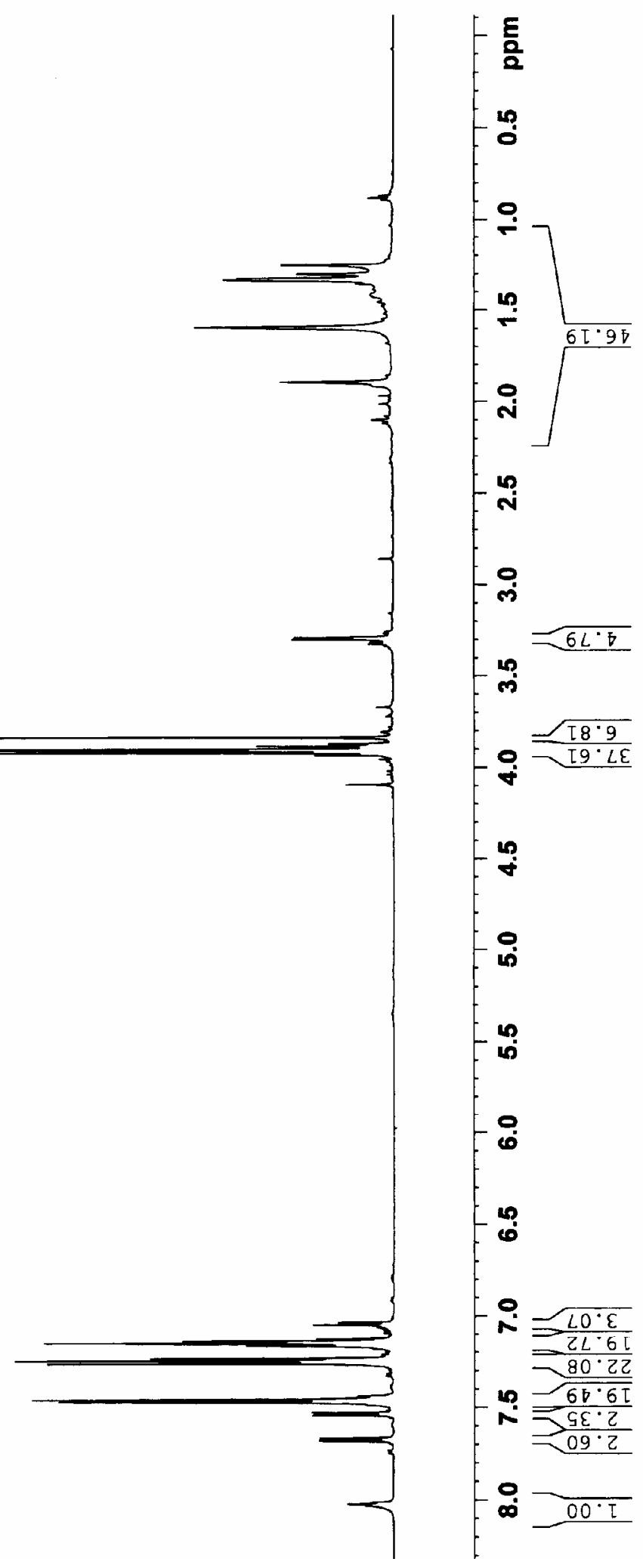




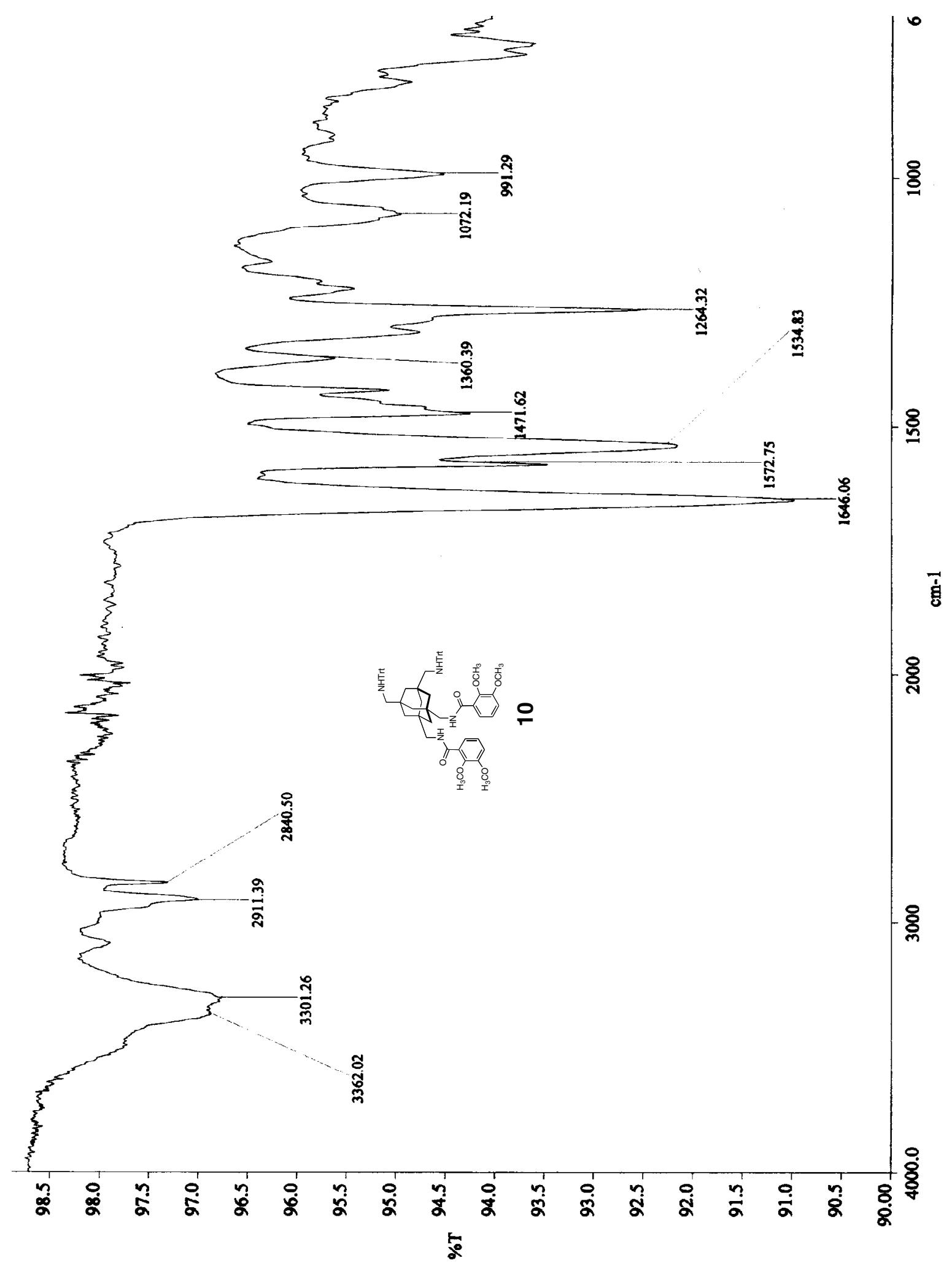

S-25 


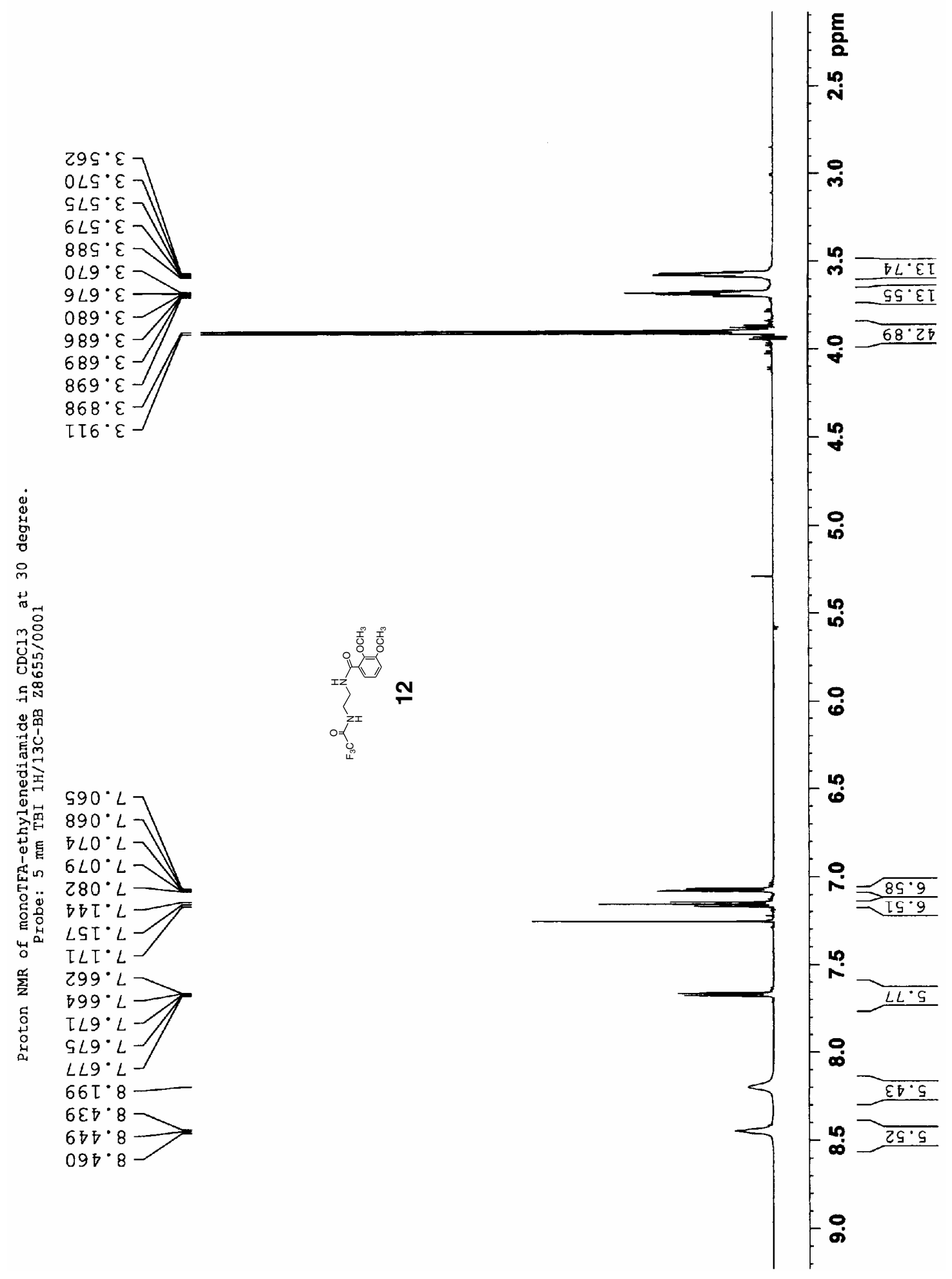




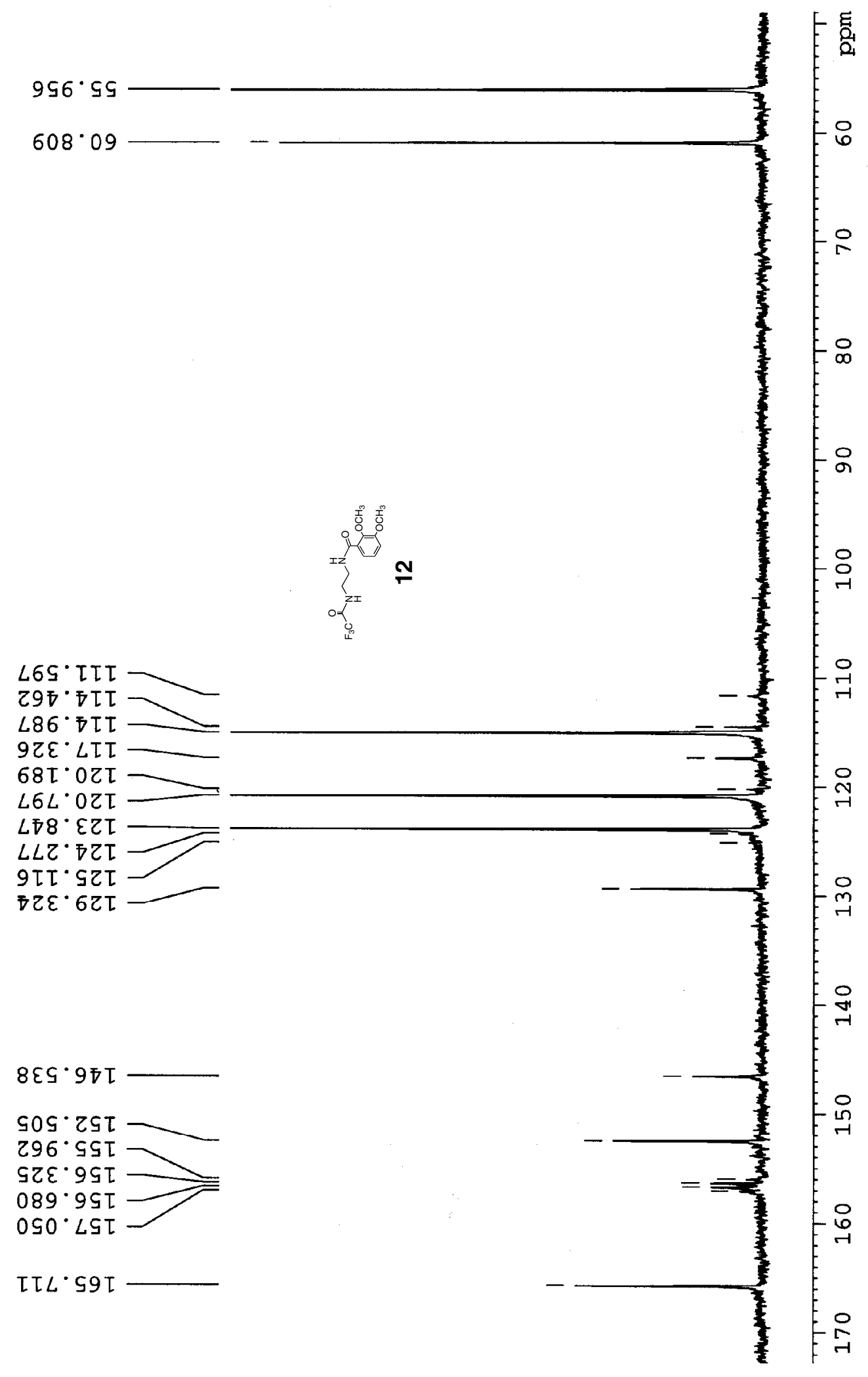




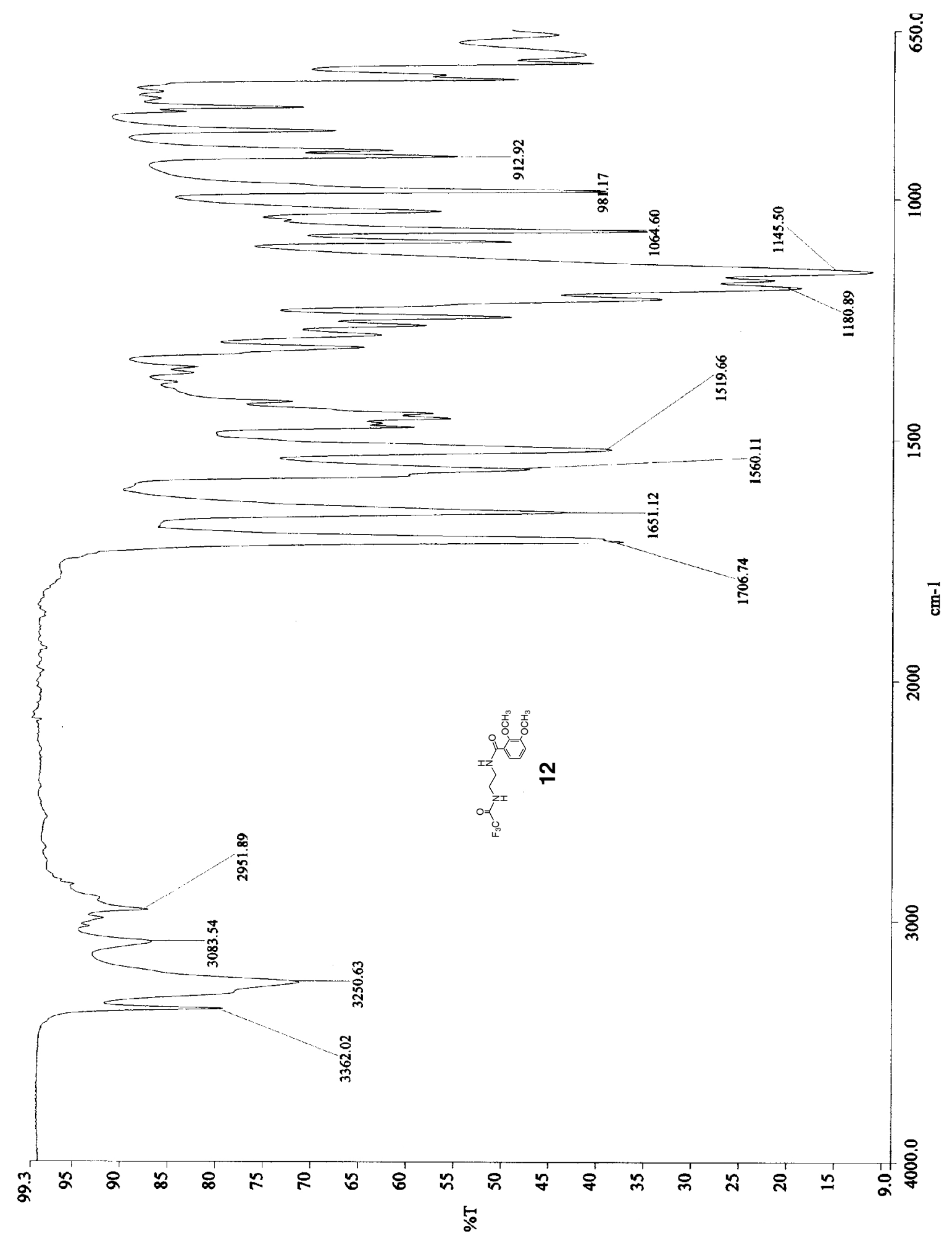



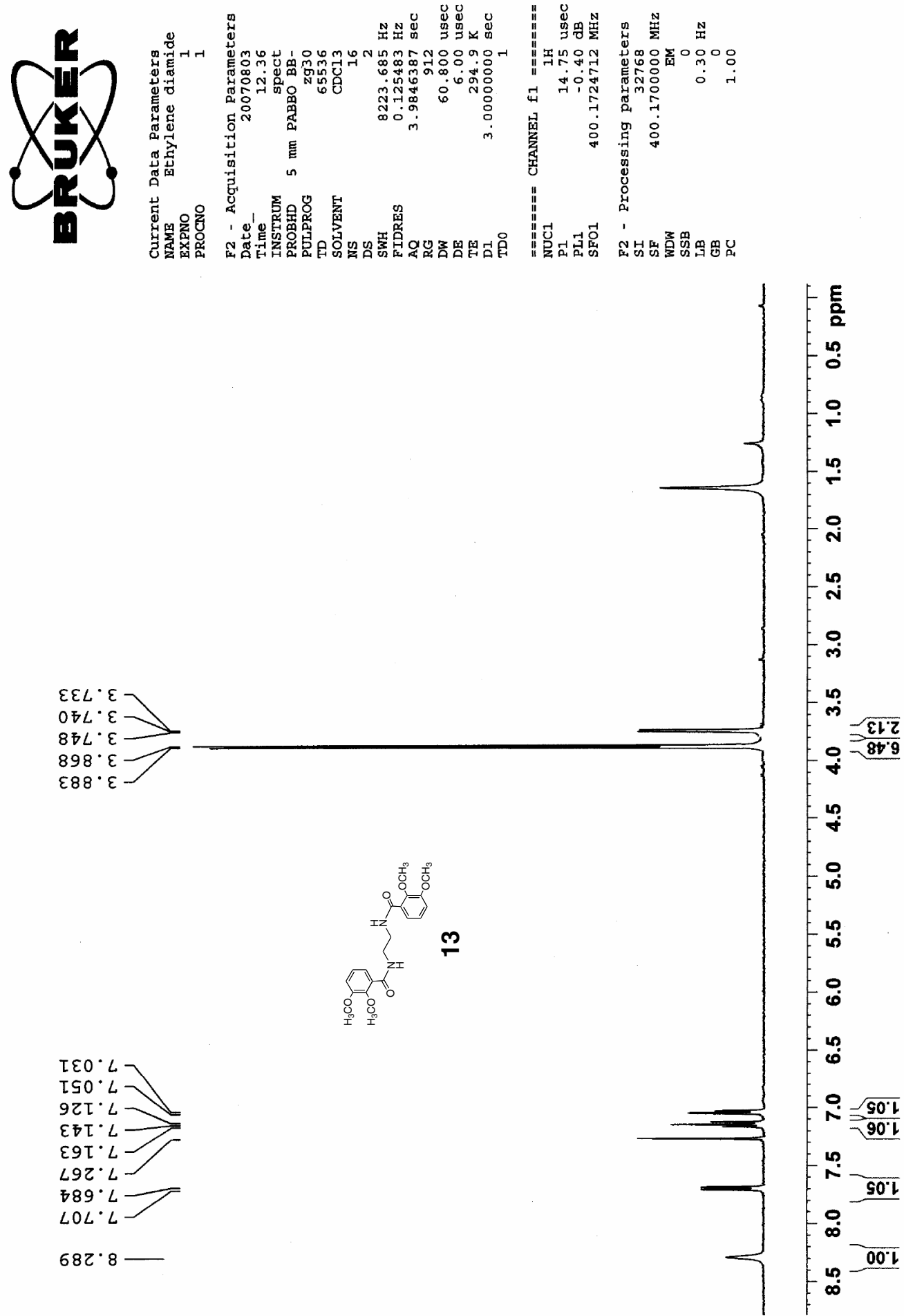


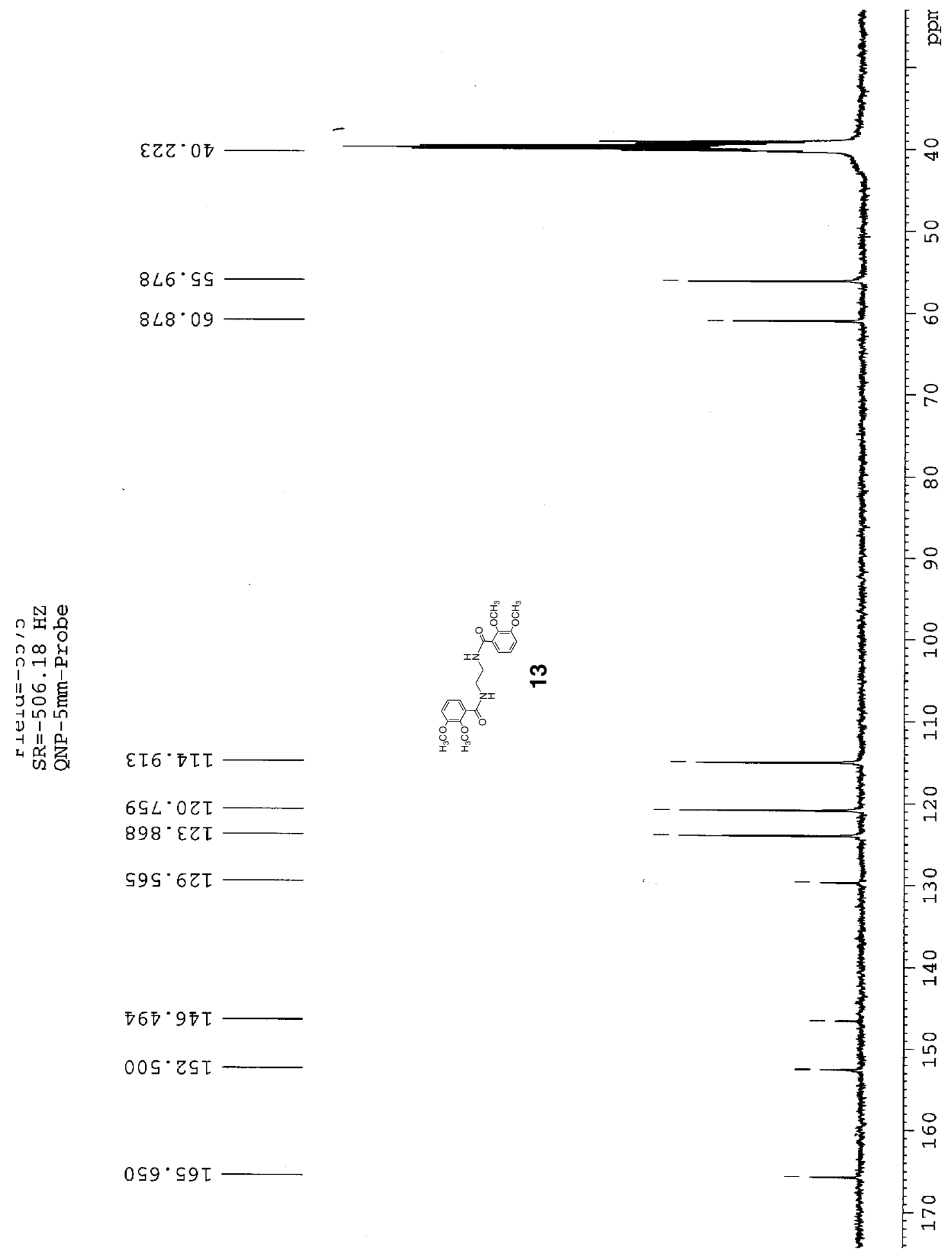




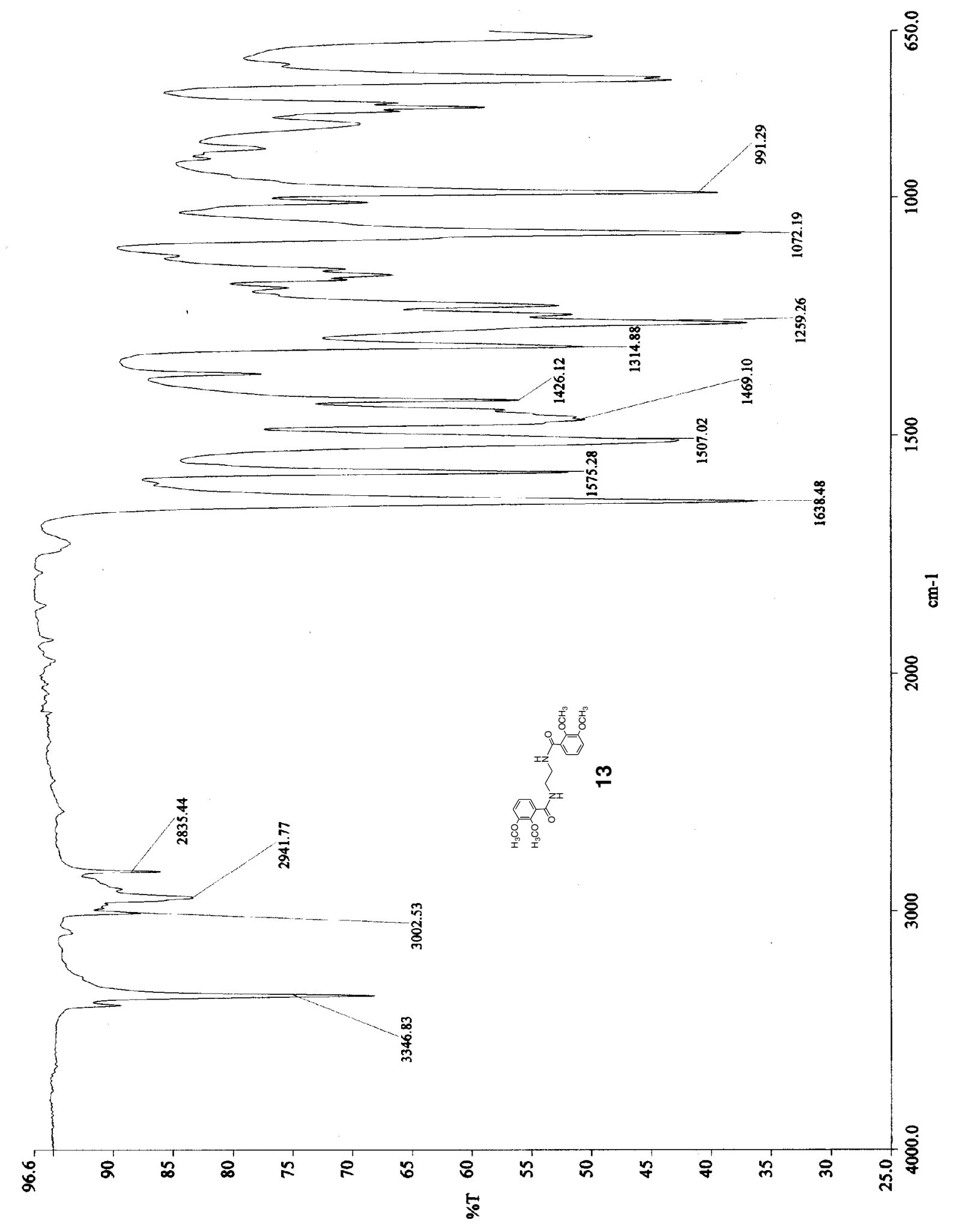

S-31 

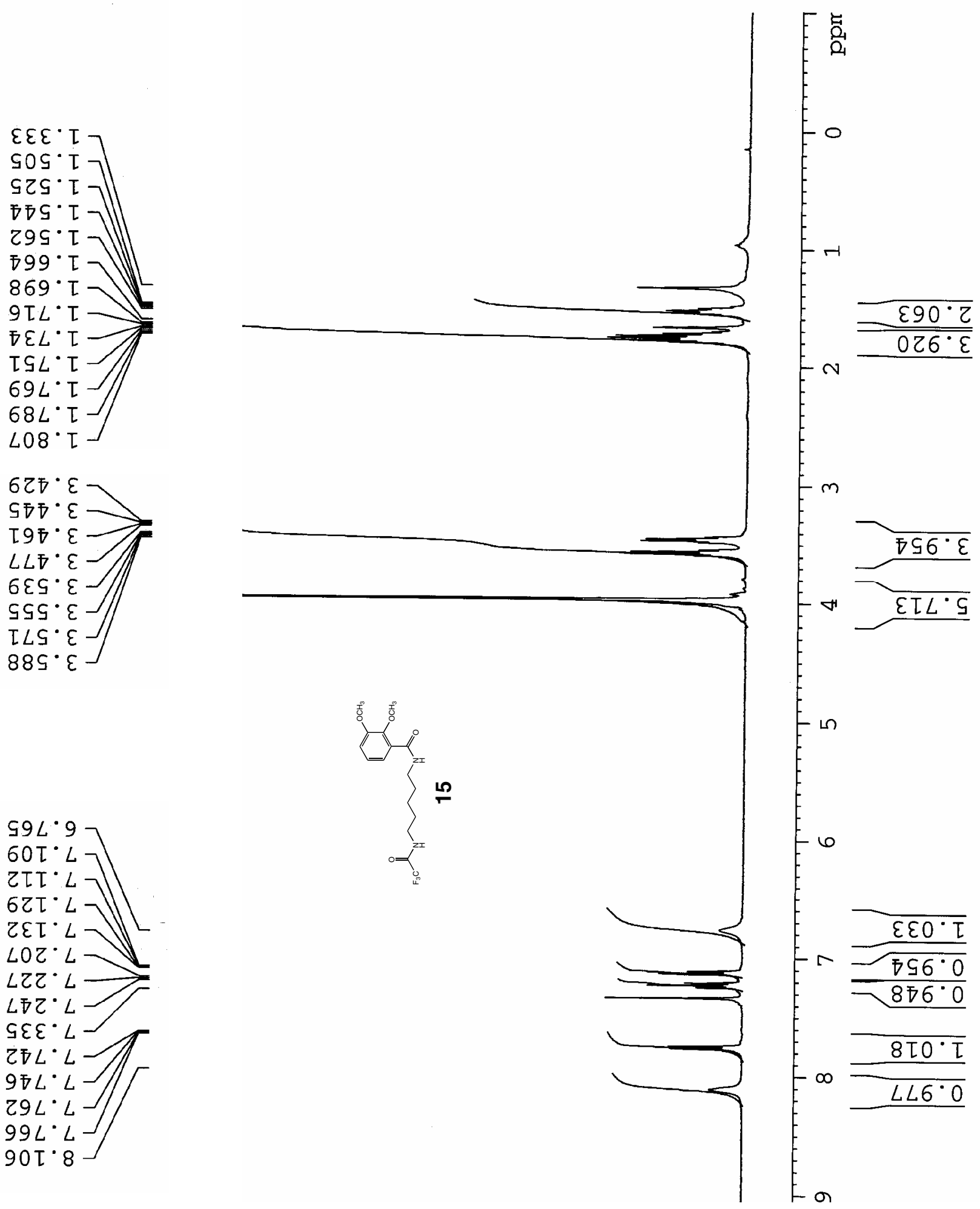


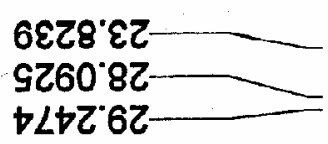

1966 $8 \varepsilon$ $988 L^{\circ} 6 \mathcal{E}$

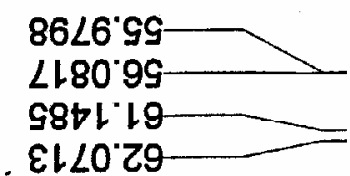

$6096^{\circ} \varepsilon$ ยงเE'ง $\angle 96 Z \angle L$ $999 \angle L L$ \&Z89'に乙L †Z8० ZZL $\angle 089^{\circ} \varepsilon 乙$ $08 Z \varepsilon^{\circ} \nabla Z$ $998 L^{\circ}+Z L$ عZ9t $9 \mathrm{Z}$

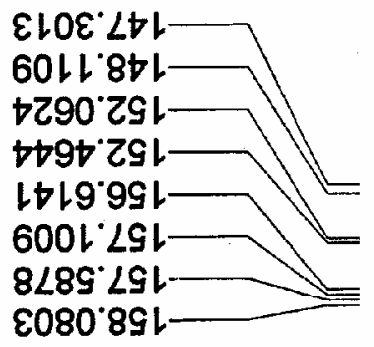
oEZt 991

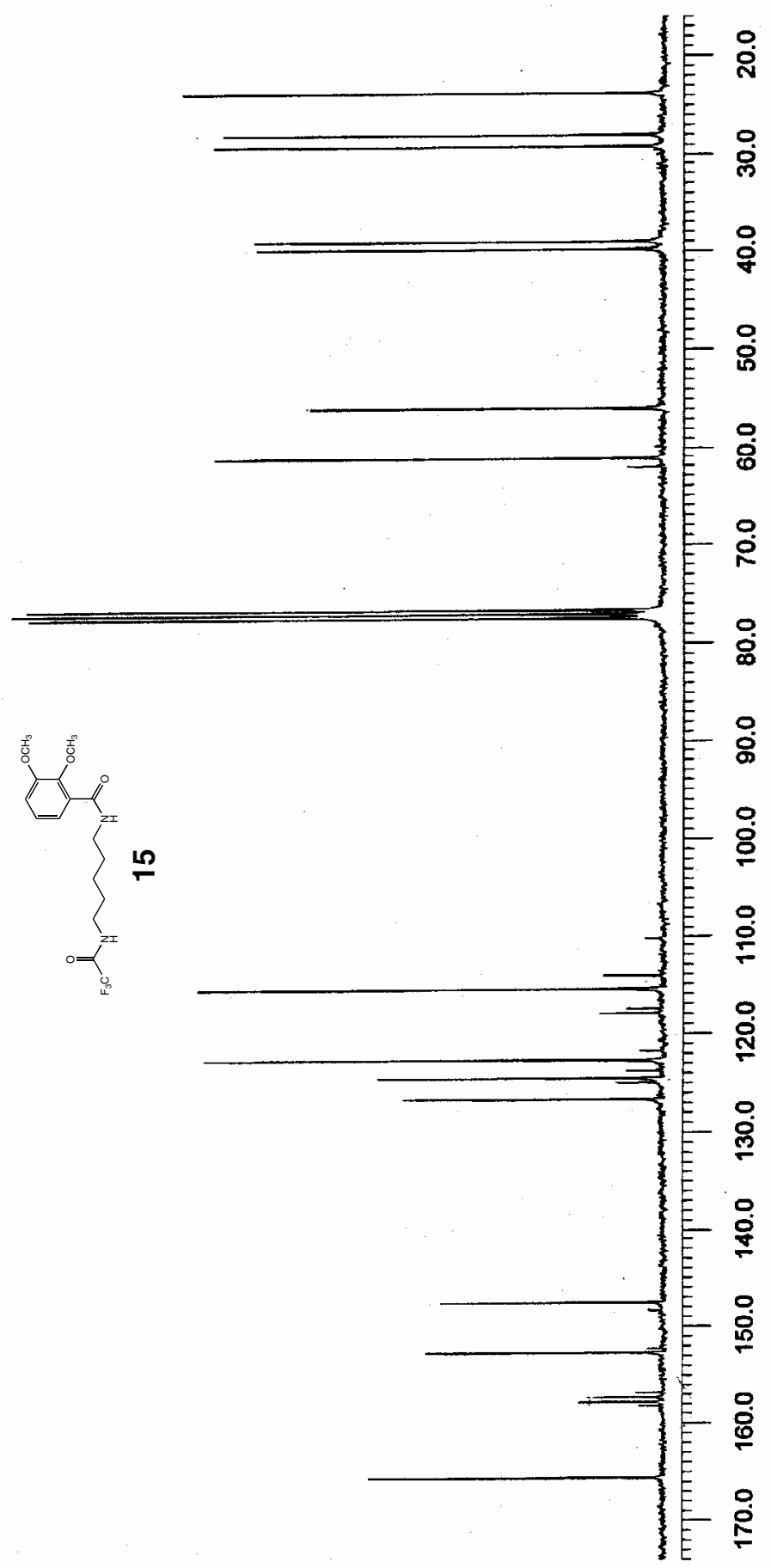




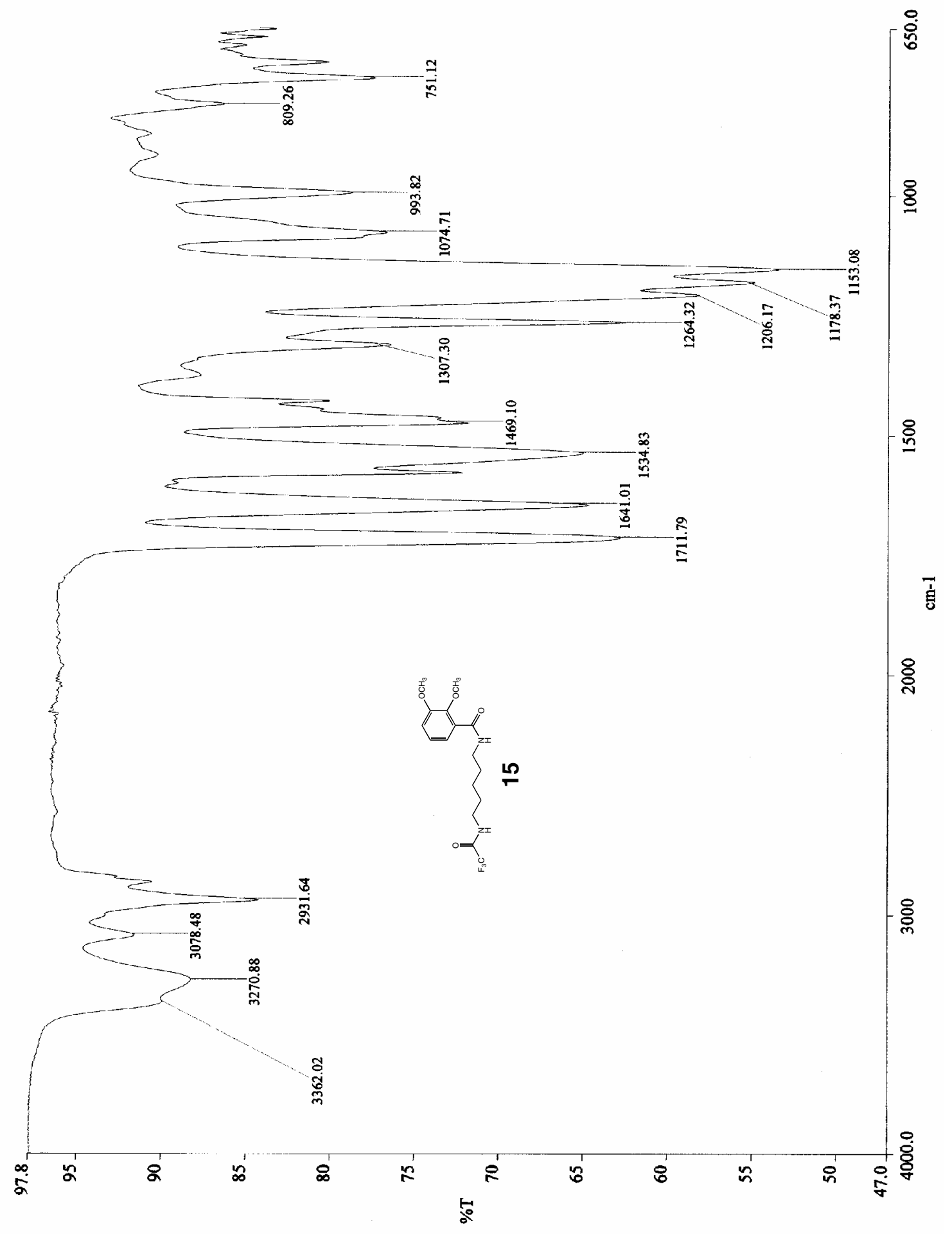




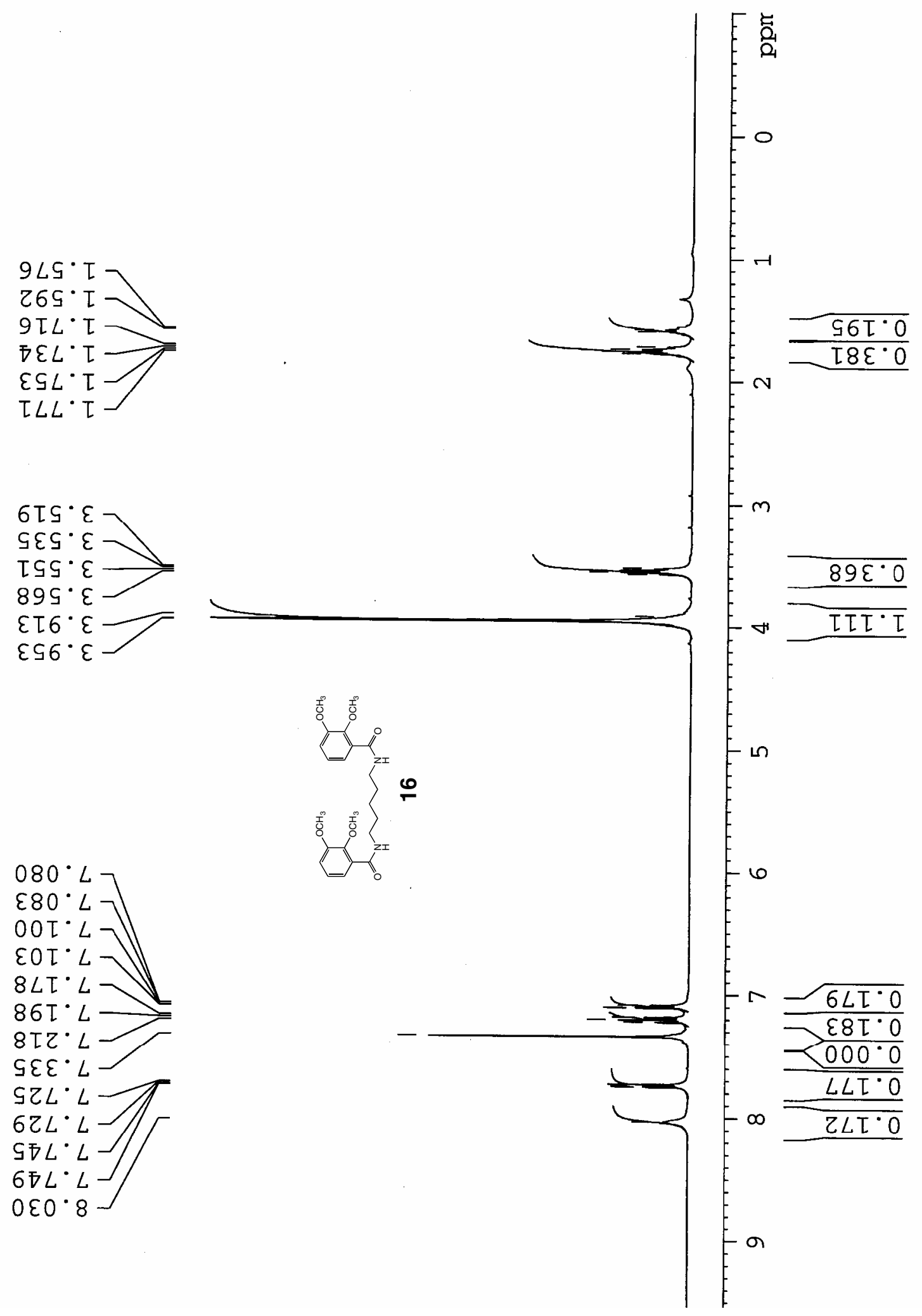




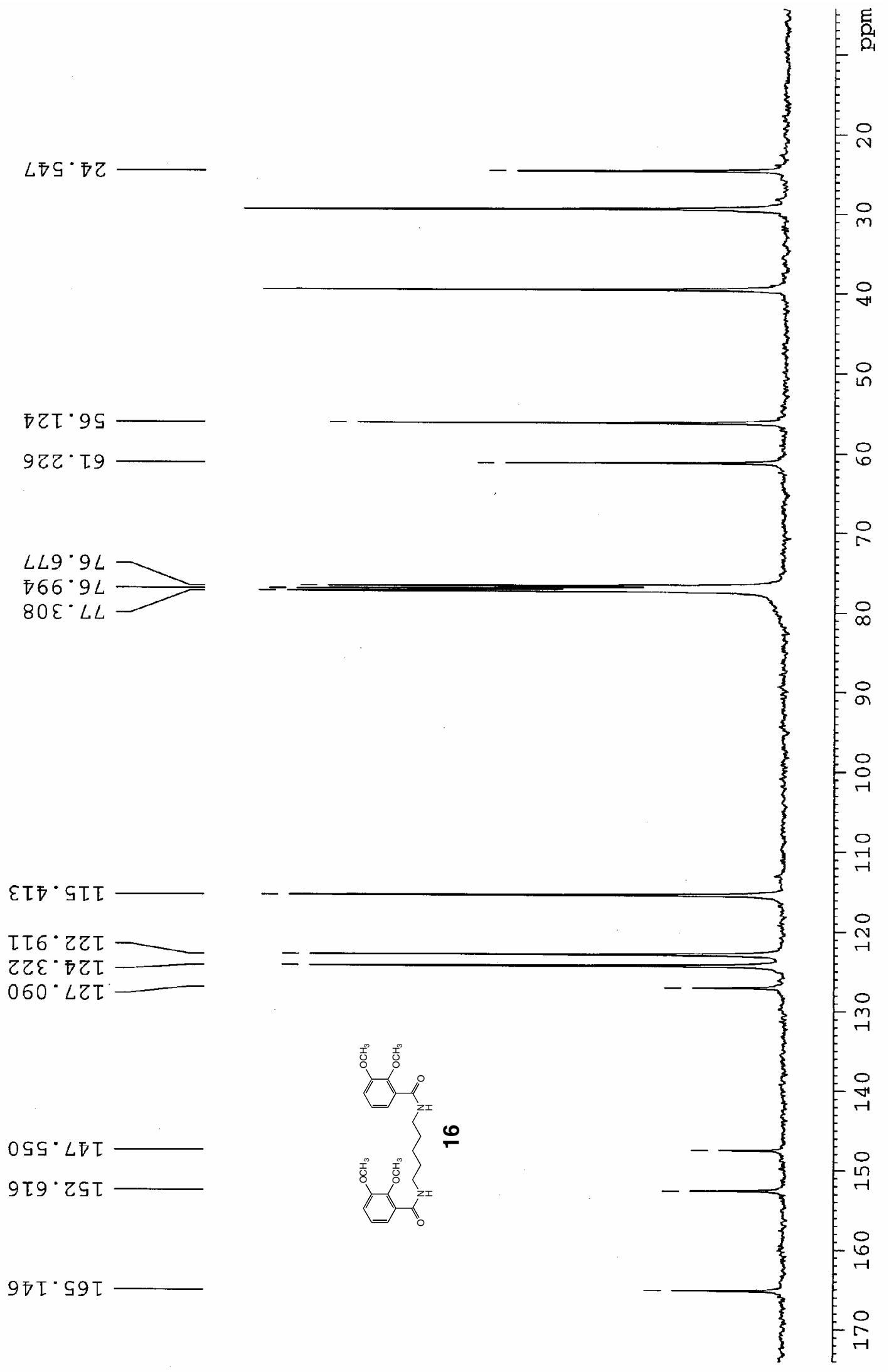




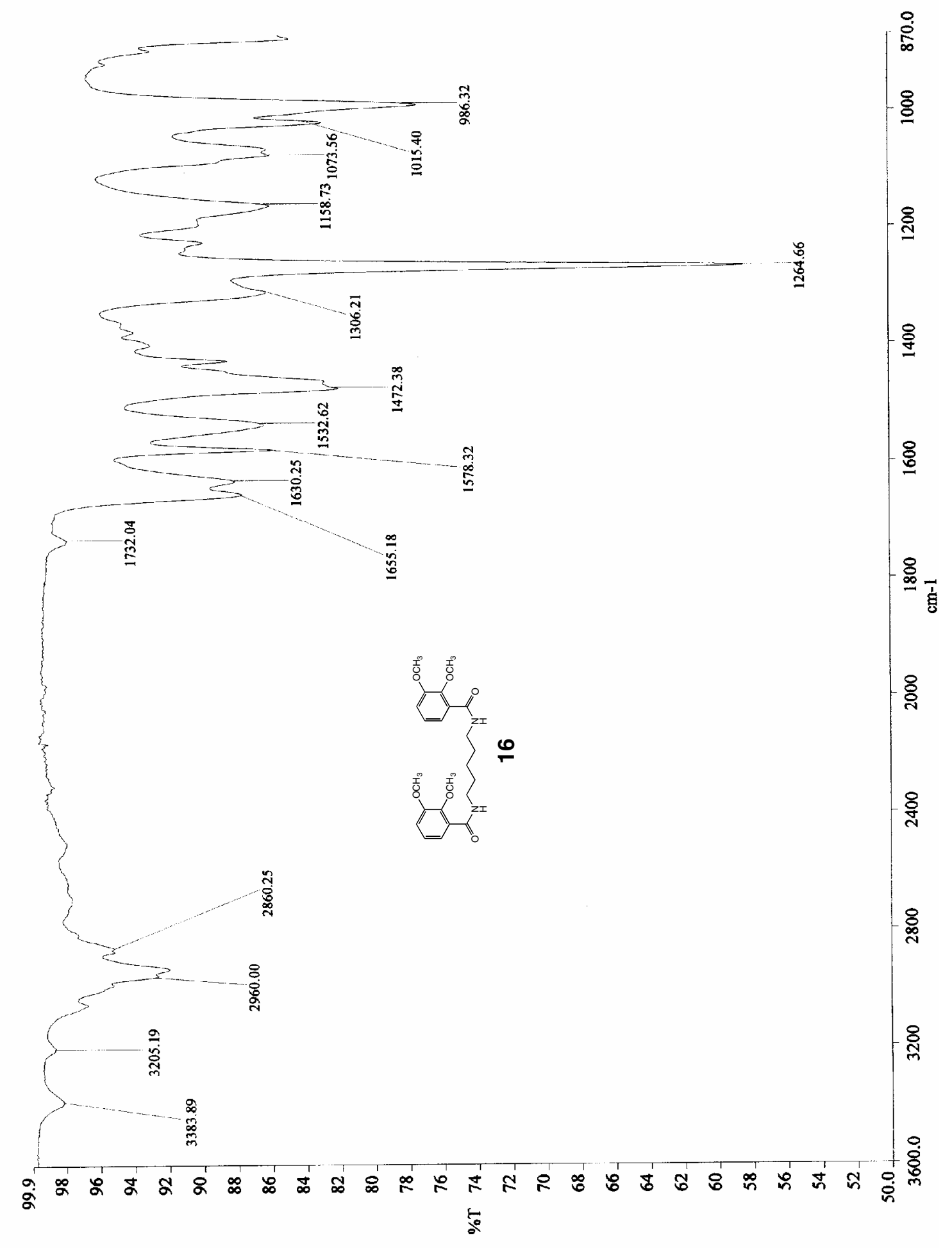



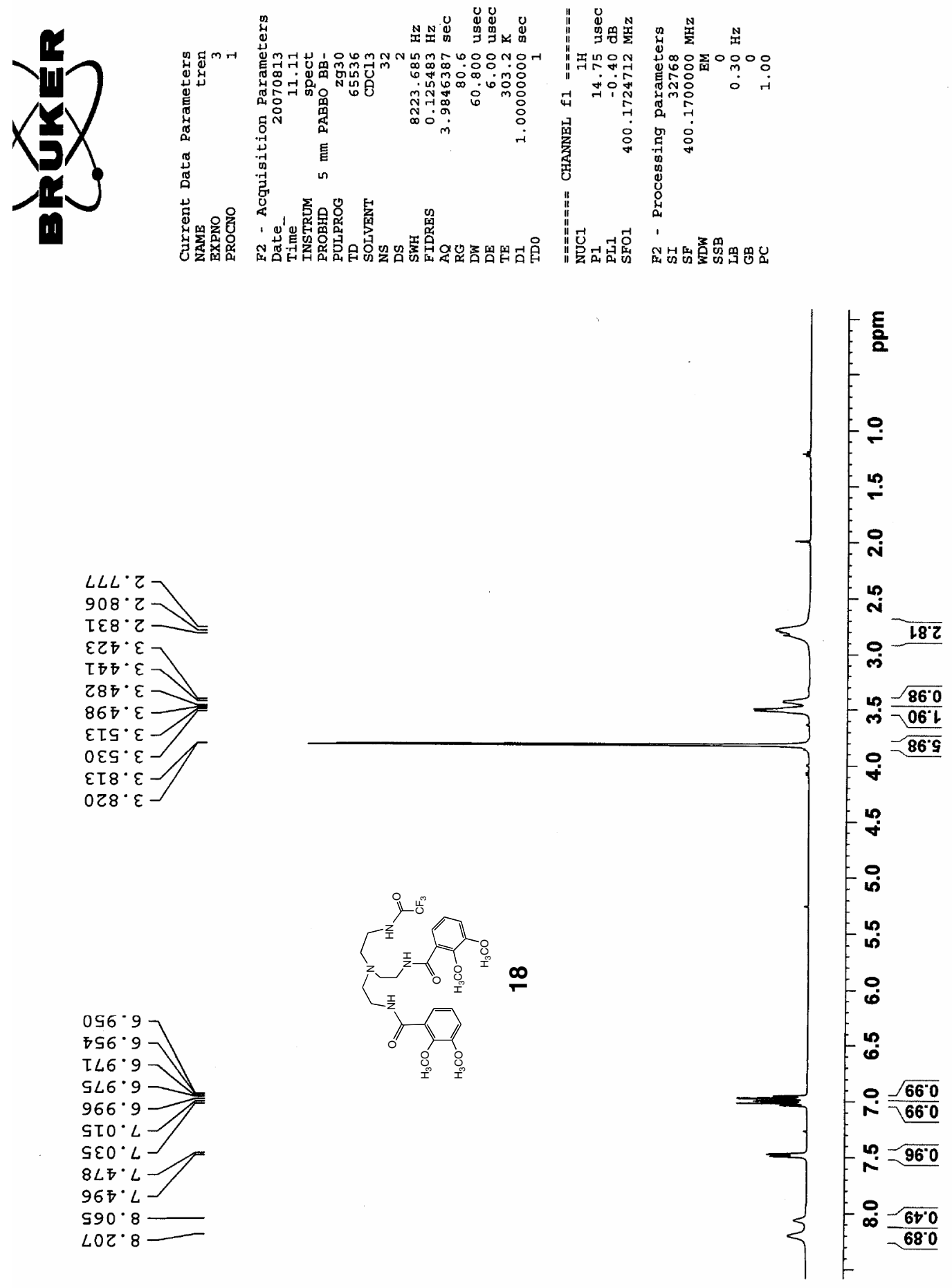


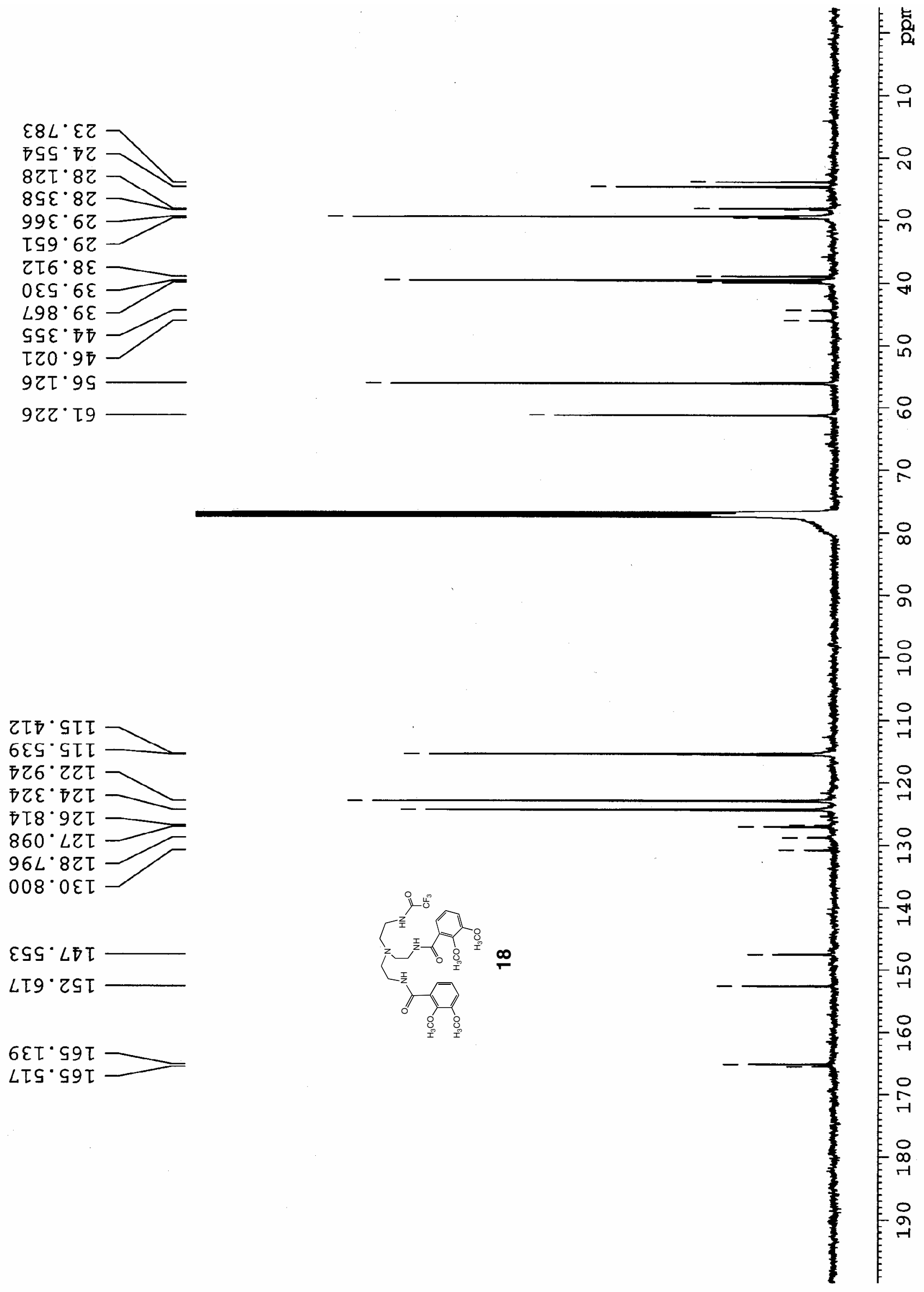




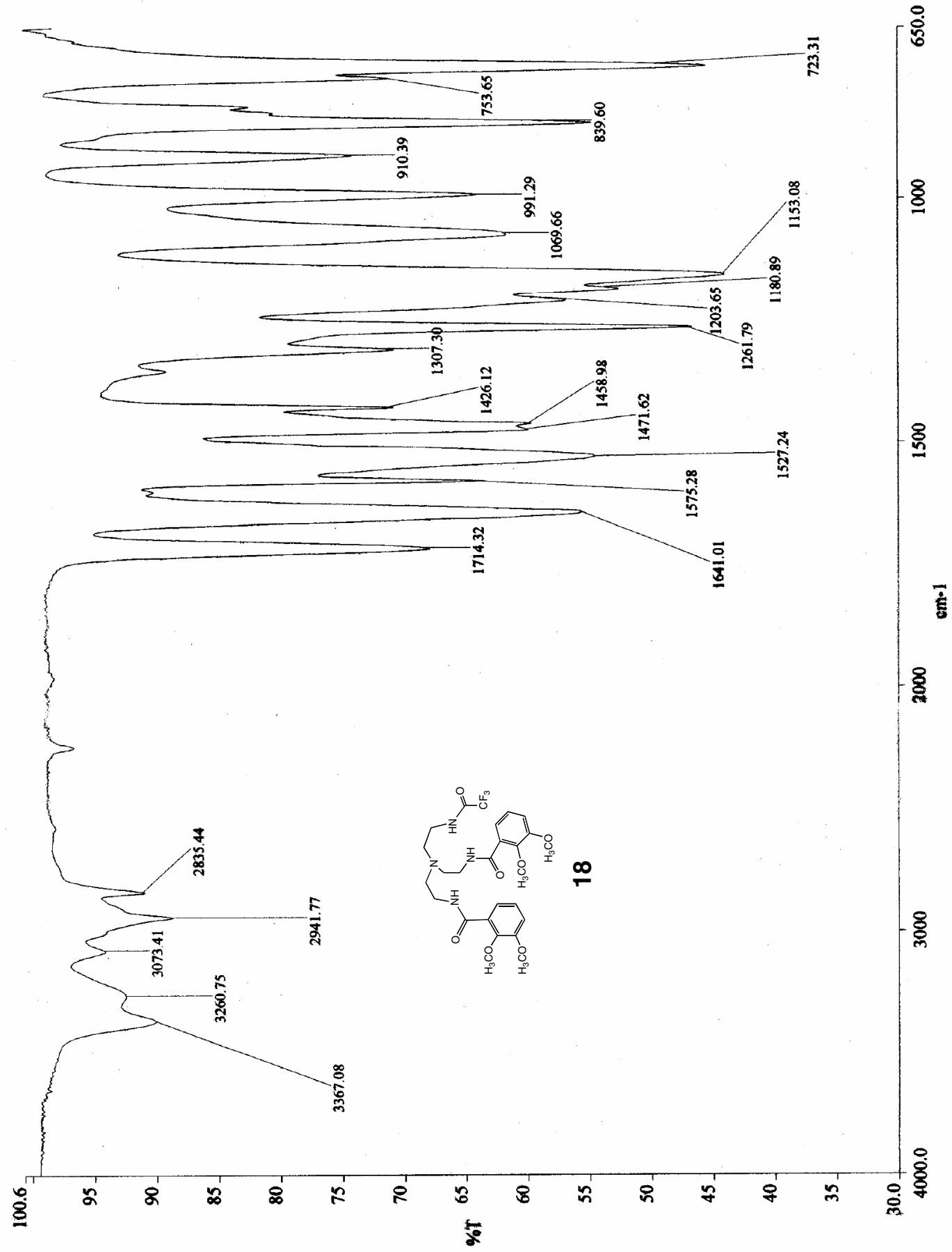



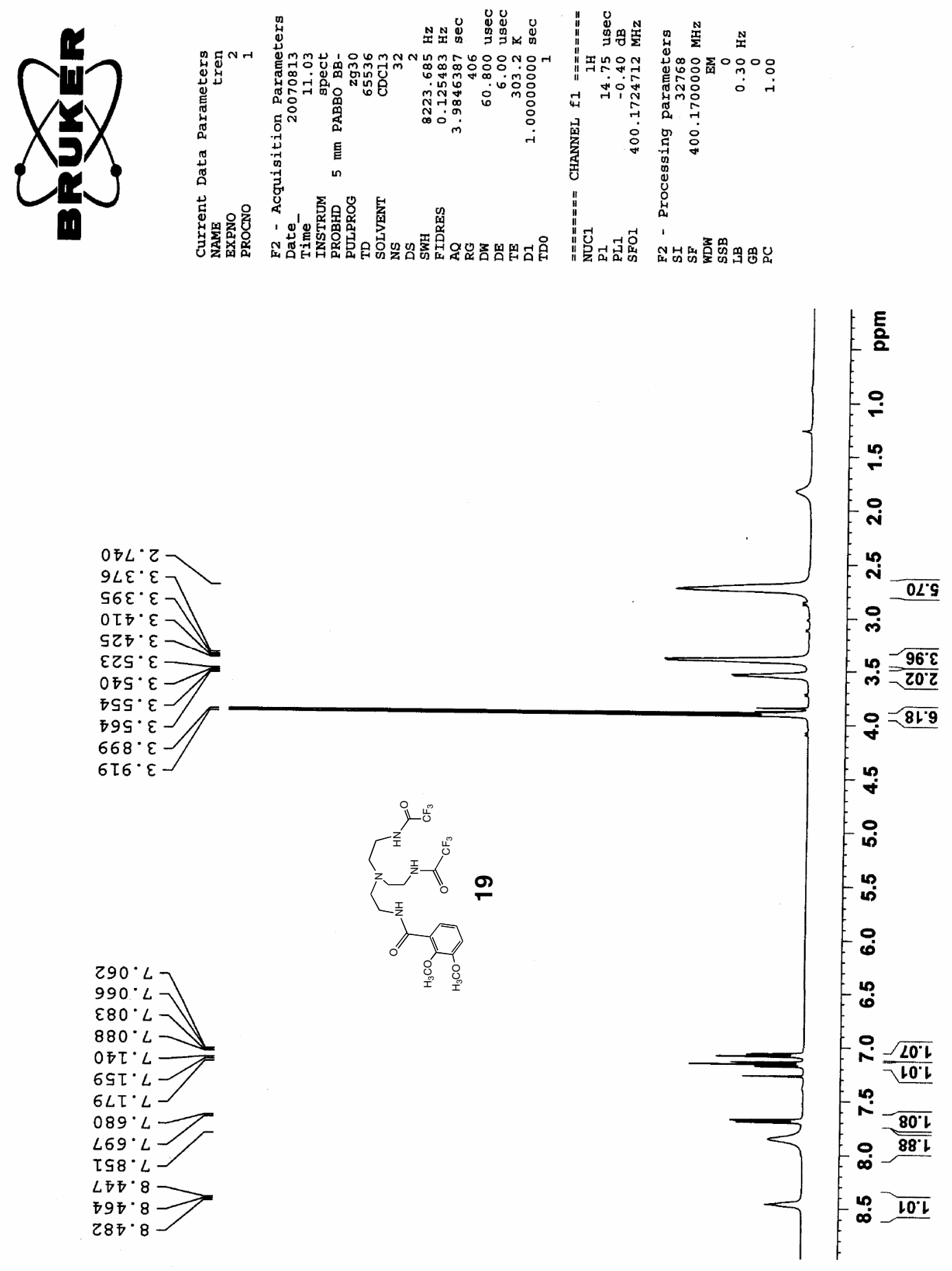

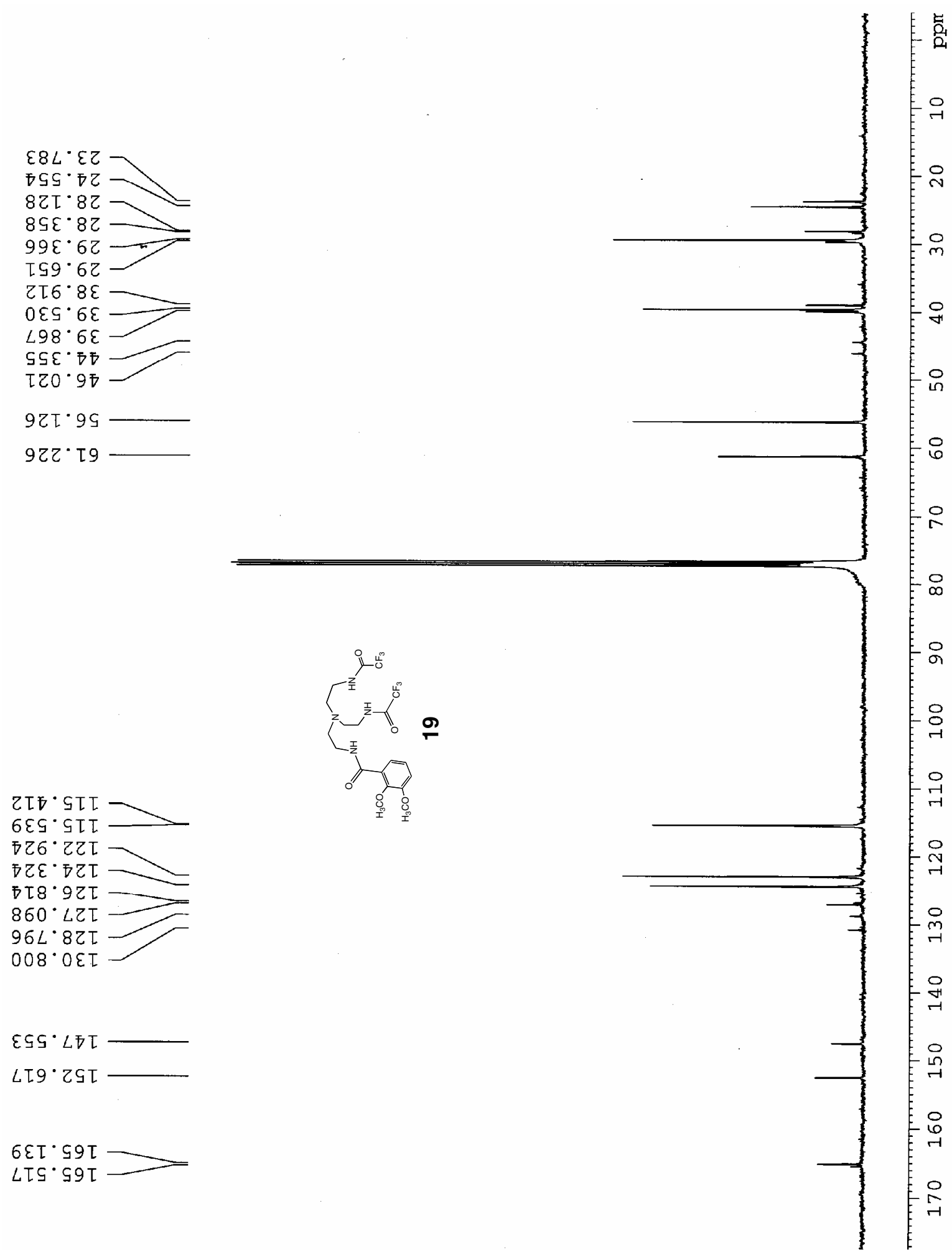


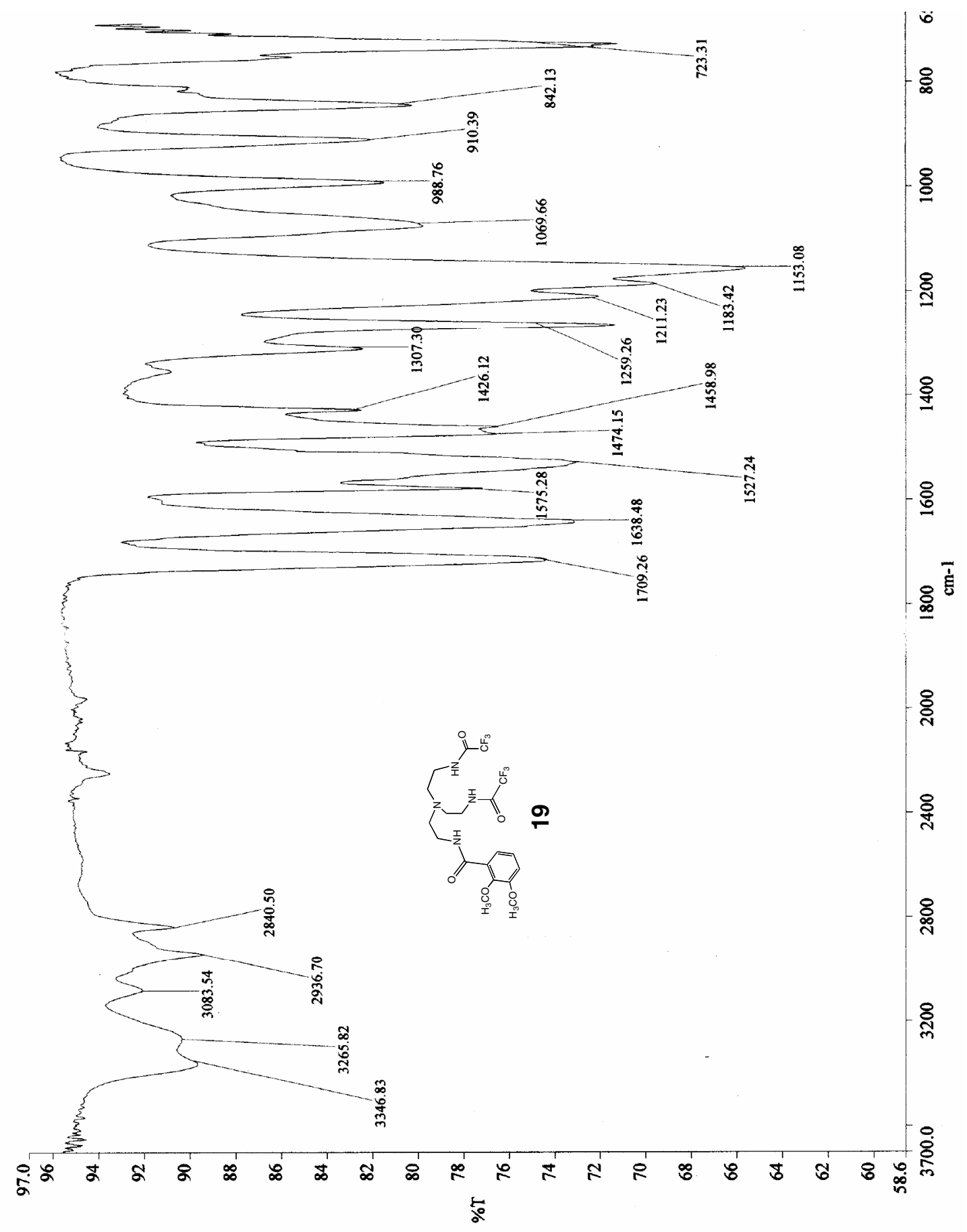




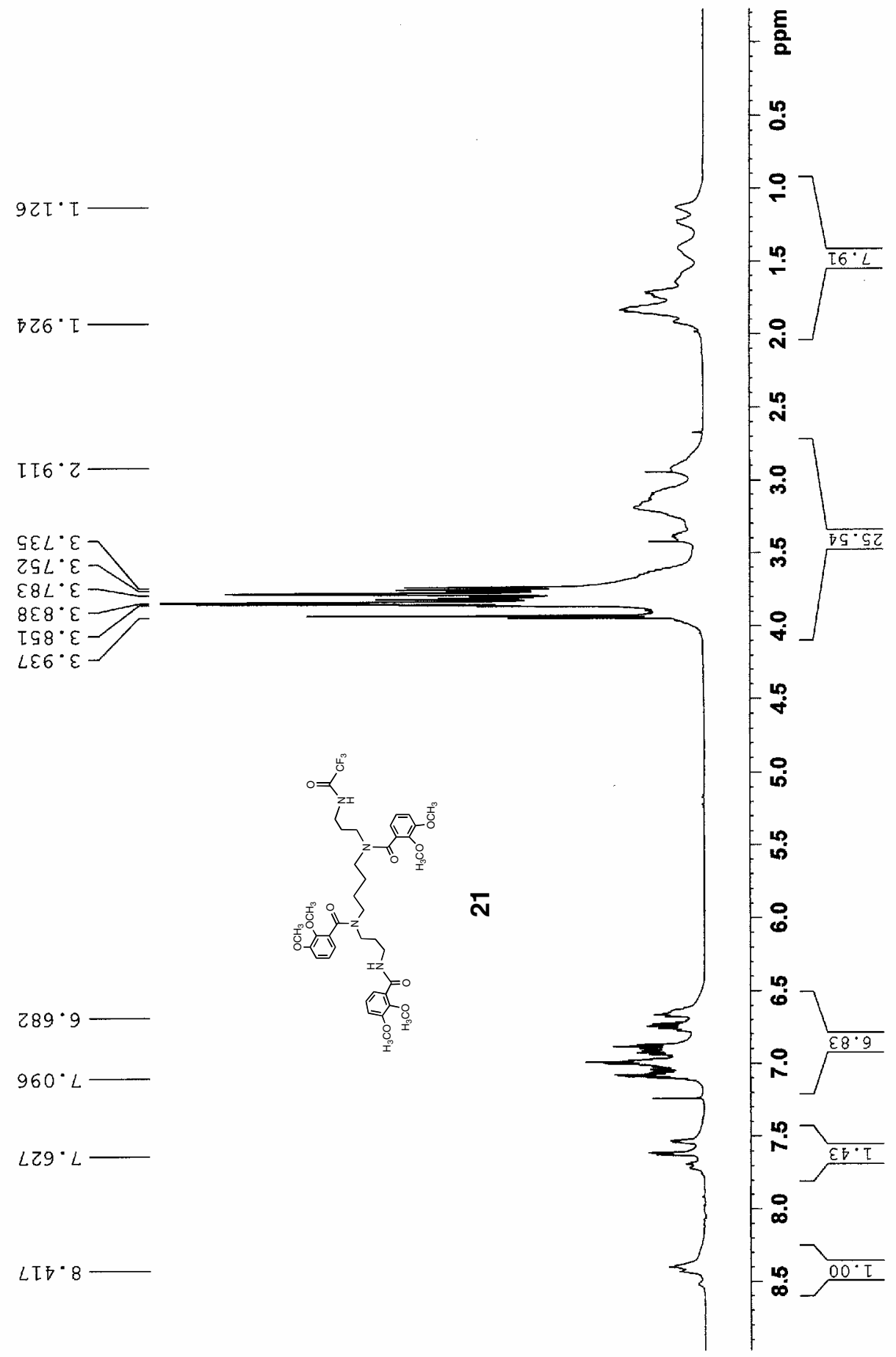



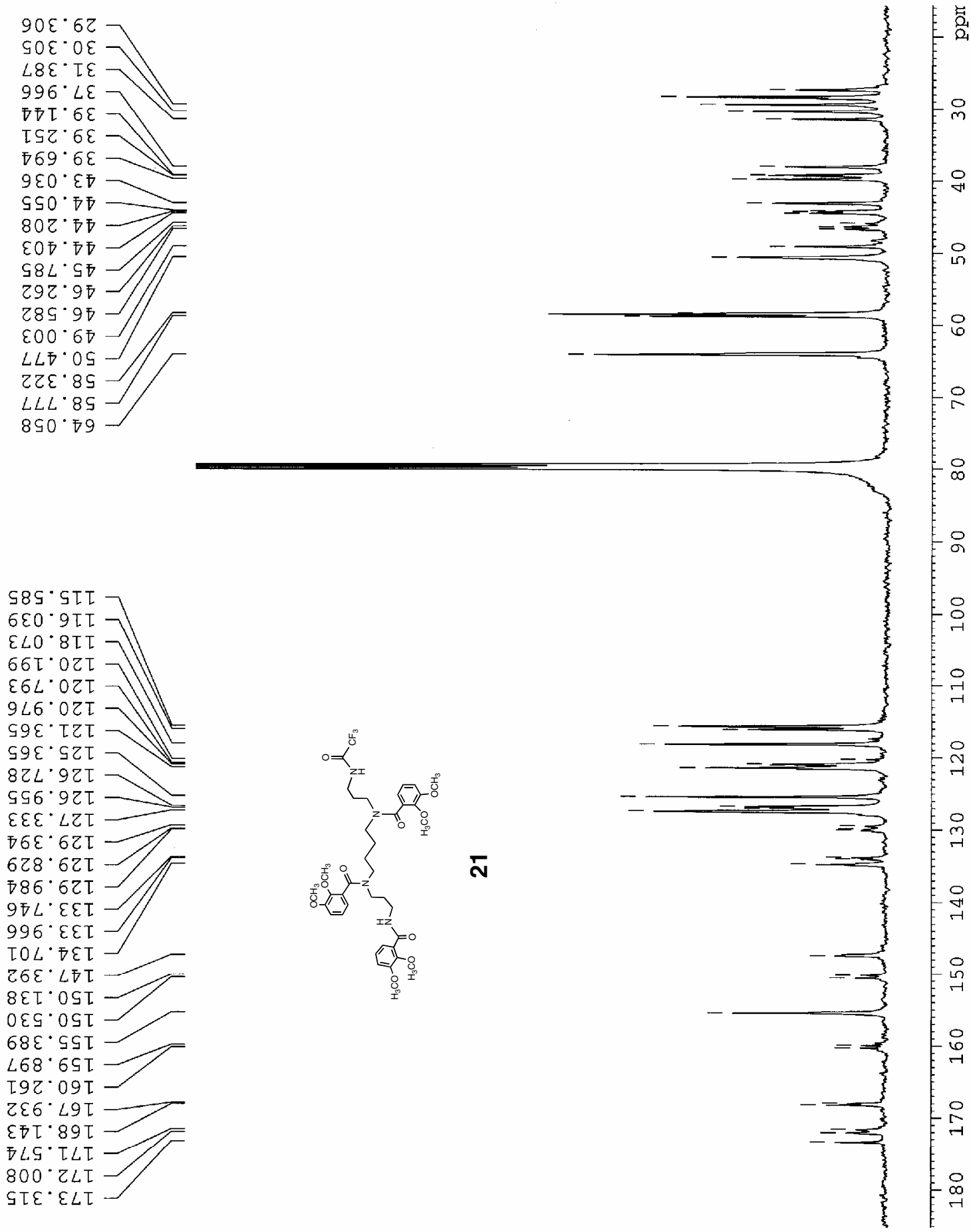


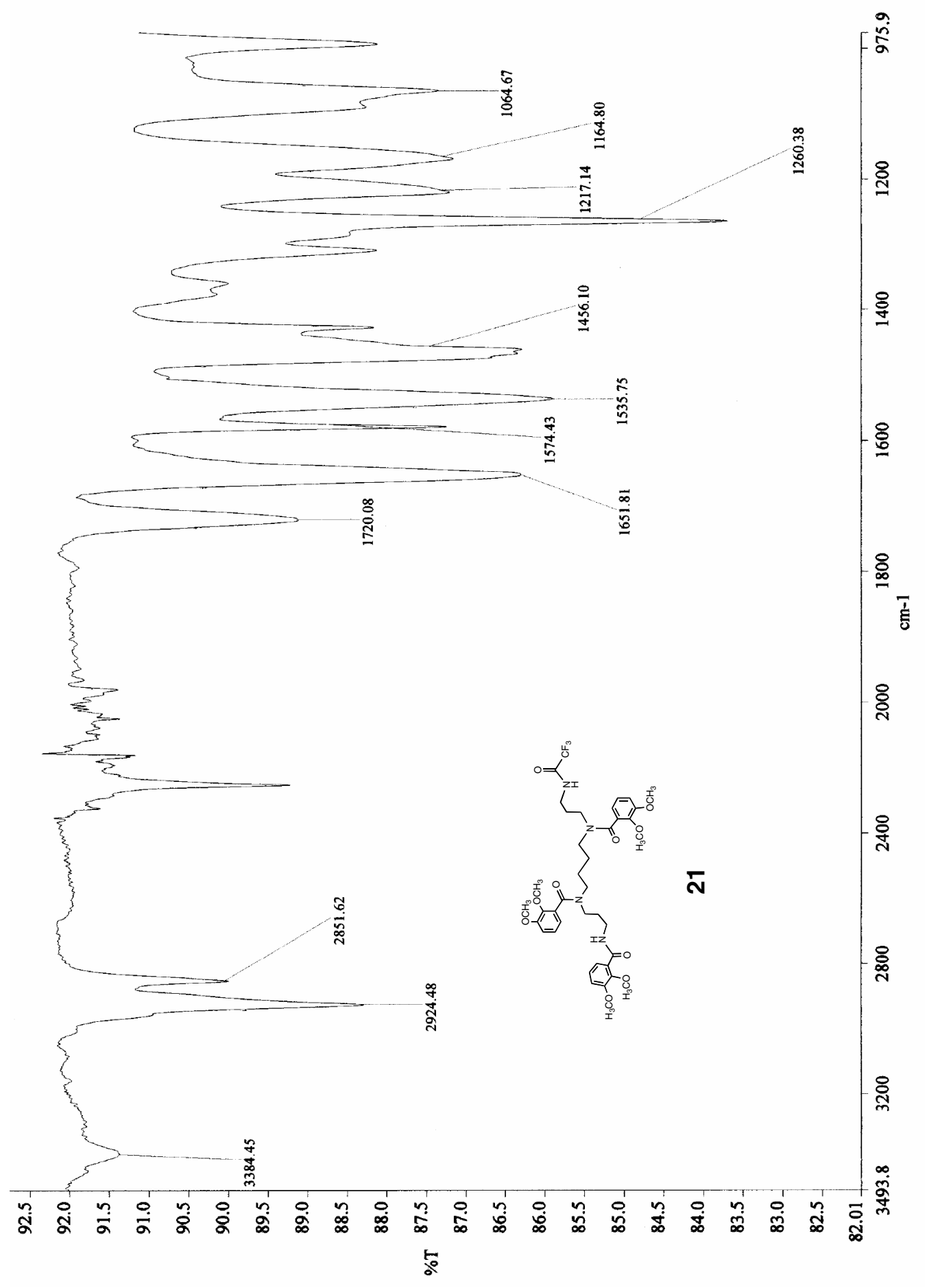



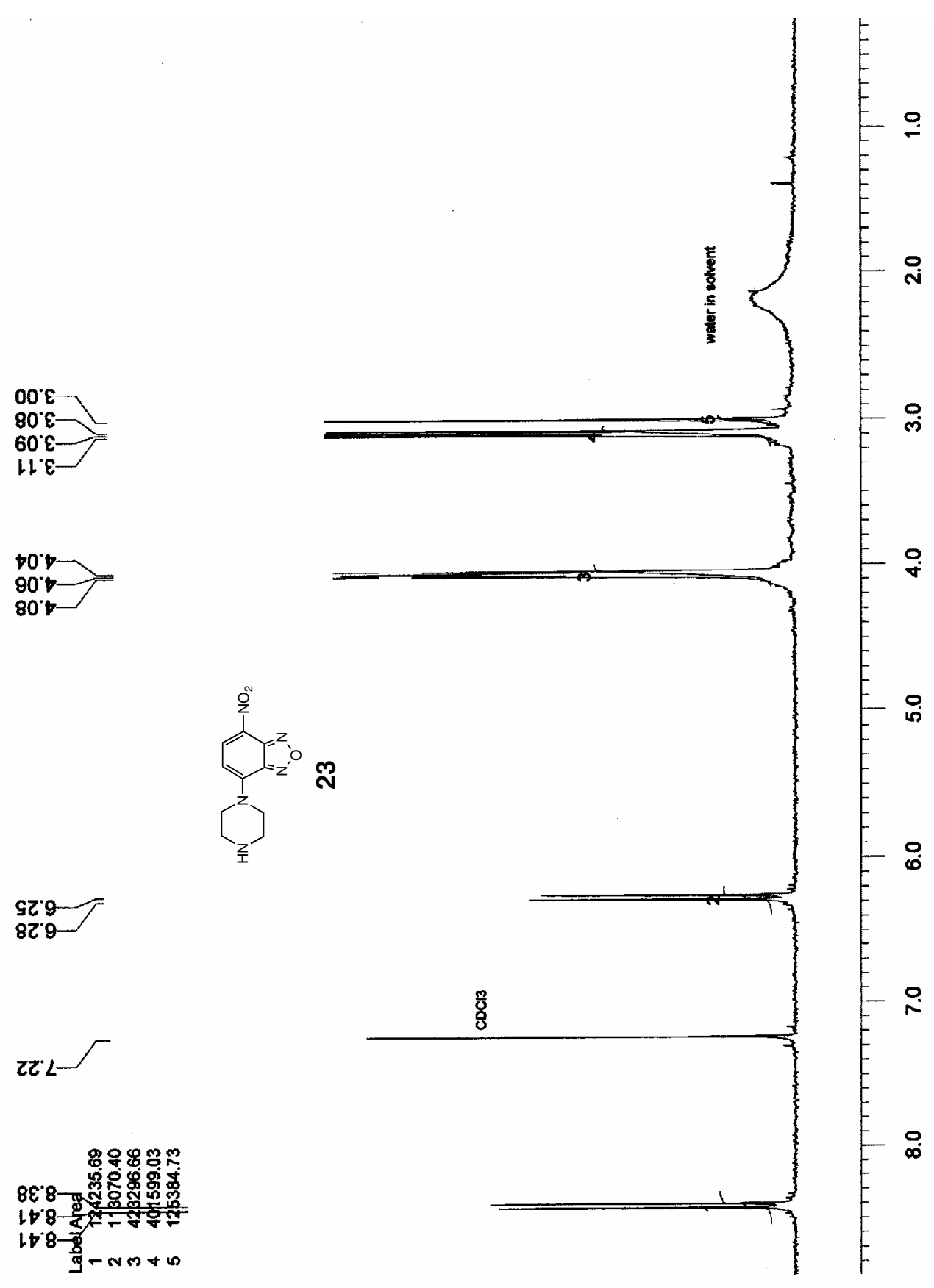


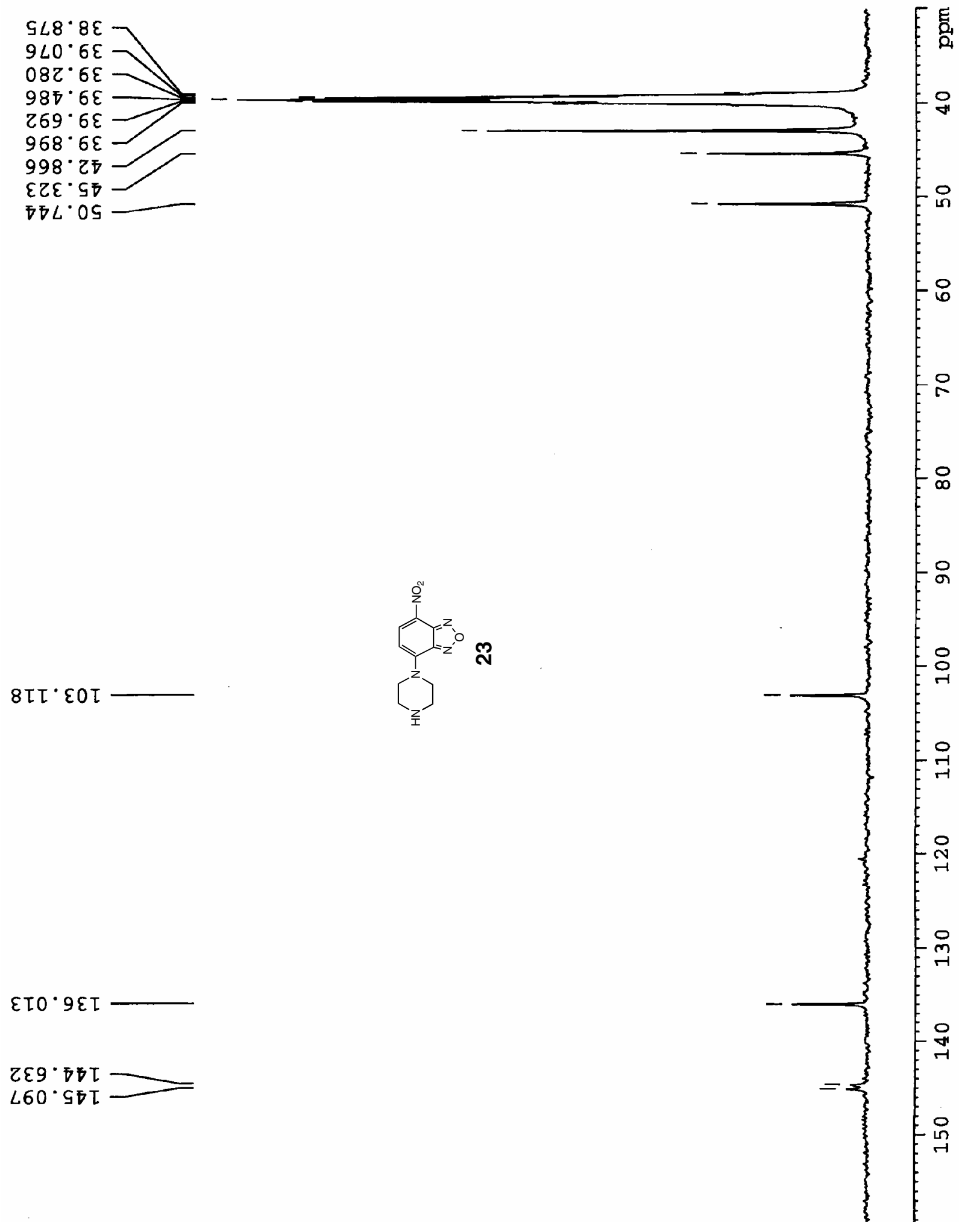




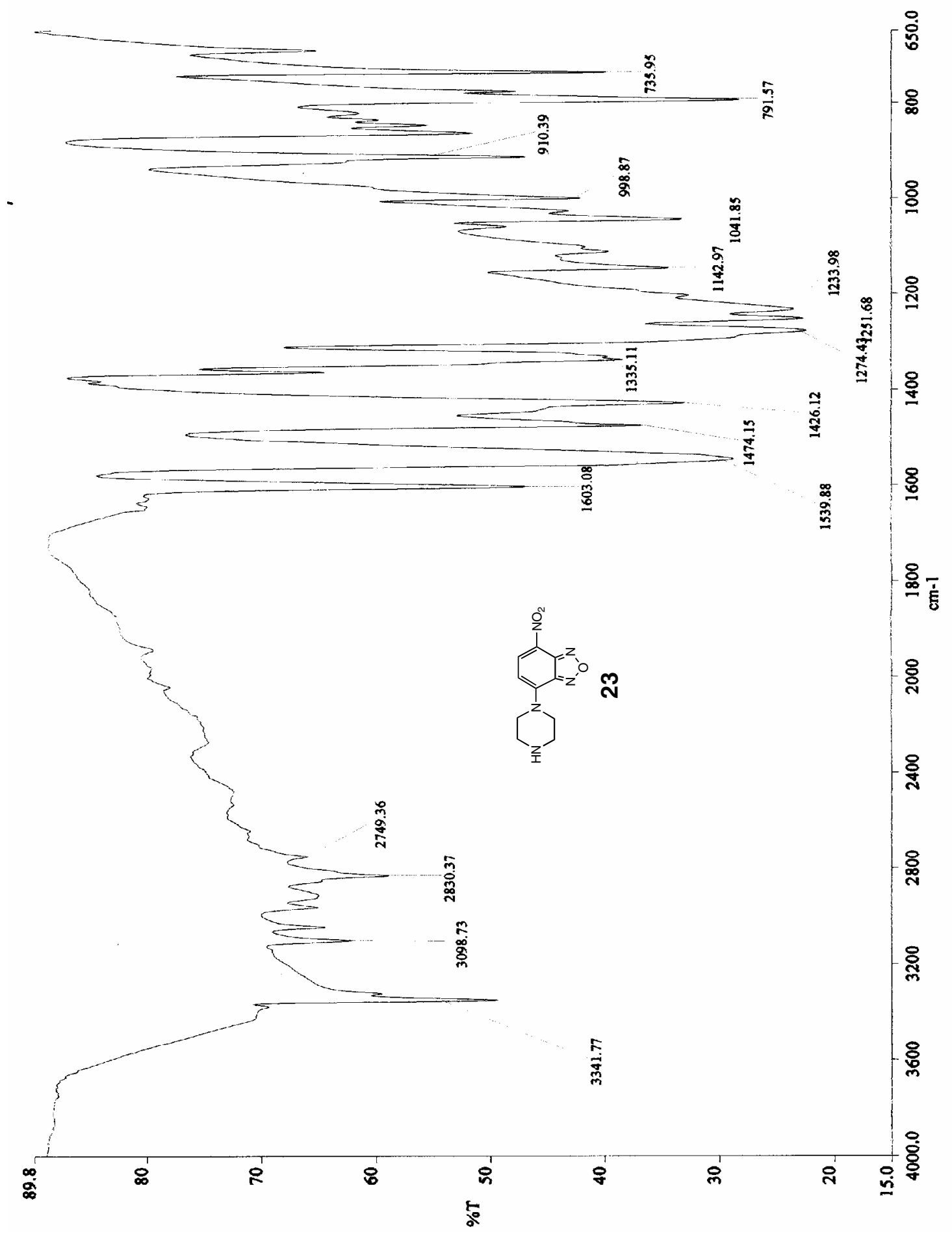

